\title{
Optimal design of fixture layouts for compliant sheet metal assemblies
}

\author{
Abolfazl Rezaei Aderiani ${ }^{1}$ (D $\cdot$ Kristina Wärmefjord $^{1} \cdot$ Rikard Söderberg $^{1} \cdot$ Lars Lindkvist $^{1} \cdot$ Björn Lindau $^{2}$
}

Received: 21 February 2020 / Accepted: 17 August 2020 / Published online: 5 September 2020

(C) The Author(s) 2020

\begin{abstract}
A preeminent factor in the geometrical quality of a compliant sheet metal assembly is the fixture layout that is utilized to perform the assembly procedure. Despite the presence of a great number of studies about the optimization of assembly fixture layouts, there is not a comprehensive algorithm to optimize all design parameters of fixture layouts for compliant sheet metal assemblies. These parameters are the location and type of hole and slot in each part, the slot orientation, and the number and location of additional clamps. This paper presents a novel optimization method that optimizes all these parameters simultaneously to maximize the geometrical quality of the assemblies. To attain this goal, compliant variation simulations of the assemblies are utilized along with evolutionary optimization algorithms. The assembly springback and contacts between parts are considered in the simulations. After determining the optimal design parameters, the optimal positions of locators are fine-tuned in another stage of optimization. Besides, a top-down design procedure is proposed for applying this method to multi-station compliant assemblies. The presented method is applied to two industrial sample cases from the automotive industry. The results evidence a significant improvement of geometrical quality by utilizing the determined fixture layout from the presented method compared with the original fixture layouts of the sample cases.
\end{abstract}

Keywords Fixture layout optimization $\cdot$ Sheet metal assembly $\cdot$ Compliant variation simulations

$\begin{array}{ll}\text { Abbreviations and nomenclature } \\ \text { BIW } & \text { Body in white } \\ \text { KPC } & \text { Key product characteristic } \\ \text { SSM } & \text { State-space modeling } \\ \text { MIC } & \text { Method of influence coefficients } \\ \text { FEM } & \text { Finite element method } \\ \text { GA } & \text { Genetic algorithm } \\ \text { RMS } & \text { Root mean square } \\ P & \text { The number of parts in the assembly } \\ Q_{p} & \text { The number of areas in part } p \\ H_{p} & \text { The number of feasible areas for hole/slot in part } \\ & p \\ \alpha_{q_{p}} & \text { Indicator of being clamped or not in area } q \text { of } \\ & \text { part } p \\ \beta_{1_{p}} & \text { The area number of the hole location in part } p\end{array}$

Abolfazl Rezaei Aderiani

aderiani@chalmers.se

1 Department of Industrial and Materials Science, Chalmers University of Technology, Gothenburg, Sweden

2 Volvo Cars, Gothenburg, Sweden

$\begin{array}{ll}\beta_{2_{p}} & \text { The area number of the slot location in part } p \\ \theta_{q} & \text { The in-plane locking direction of a slot } \\ \mathbf{N} & \text { Normal vector } \\ \mathbf{d} & \quad \text { Vector of in-plane locking direction of a slot in } \\ & \begin{array}{l}\text { slot's local coordinate system } \\ \mathbf{d}^{\prime}\end{array} \\ & \begin{array}{l}\text { Vector of in-plane locking direction of a slot in } \\ \text { the global coordinate system }\end{array} \\ \mathbf{Q} & \text { Transformation matrix } \\ F_{b} & \text { Clamping forces before welding } \\ F_{a} & \text { Clamping forces after welding } \\ D_{a} & \text { Deviations after releasing the clamps } \\ D_{b} & \text { Deviations before clamping } \\ K_{a} & \text { Stiffness matrix of assembly after welding } \\ K_{b} & \text { Stiffness matrix of the assembly before welding } \\ S & \text { Sensitivity matrix of assembly } \\ W & \text { Jacobian matrix of rigid body motions constraint }\end{array}$

\section{Introduction}

Geometrical variations are inevitable consequences of mass production. These variations can cause both aesthetic and functional problems in addition to the combined quality cost of repairs, reworks, scraps, etc., that they impose on the 
production. Consequently, 10 to $40 \%$ of the profit in the automotive industry is being lost due to the geometrical variations [1].

Spot-welded sheet metal assemblies are the dominant types of assemblies in the automotive industry [2]. To fabricate these assemblies, the sheet metal parts are fixed in an assembly fixture. Then, the spot welding is performed and the assembly is released from the fixture. At this stage, springback can occur in the assembly because of implied stresses during the clamping and welding procedures. This springback and accordingly the geometrical quality of the assembly can be predicted by compliant variation simulation of the assembly procedure.

Geometrical variation of a sheet metal assembly is affected by several factors, including part variations, fixture layout, tool variations, weld sequence, etc. Part variations are not completely avoidable because of the limited accuracy and precision of production tools and relatively higher costs of producing more accurate and precise parts. A fixture layout, however, is a completely designable factor that can amplify or lessen the effects of part variations in the final assembly.

Section 1.1 introduces fixture layout and related terms briefly. Thereafter, Section 1.2 presents a literature review on assembly fixture layout optimization. Subsequently, the research gap and scope of this paper in addressing it are illustrated in Section 1.3.

\subsection{Fixture layout}

A fixture layout should fixate at least six degrees of freedom of a compliant part by utilizing different types of locators. To achieve this goal, different arrangements of locators can be utilized. Together, the locators of a part constitute a locating scheme [3]. If the part is a compliant sheet metal, which applies to the most of sheet metals in body in white (BIW), some supports in means of clamping units can be utilized to over-constrain the part to withstand the resulting deformations from external and process forces. Consequently, the corresponding locating schemes will be $\mathrm{N}-2-1$ in which the number of additional clamps is $\mathrm{N}-3$.

Production of spot-welded sheet metal assemblies requires fixtures for two different applications. The first application is positioning and welding the parts (or sub-assemblies). The fixtures that are utilized for this application are referred to as assembly fixtures. In assembly fixtures, the goal is to have the maximum robustness so that the effects of variations of parts and tools are minimal in assembly variations [4]. The second application is to position the assembly for measuring its key product characteristics (KPC) after the assembly process is performed. The locating positions of these fixtures are datum positions and the utilized fixtures for this application are referred to as inspection fixture or measurement fixtures.

In multi-station assemblies, the measurement fixture layout (datum positions) in a station is usually defined based on the assembly fixture layout for that assembly in the next station. Accordingly, the fixture layouts in these assemblies cannot be designed independently from other stations. To avoid variation propagation in these assemblies, the locations of locators in the subsequent stations should be selected from their locations in the preceding stations [5].

There are different types of locators in assembly fixtures, including 4ways-pins, 2ways-pins, and NC-blocks. Each part has a hole where a 4 ways-pin is positioned and a slot for 2 ways-pins. Therefore, having a hole and a slot in this paper refers to having a 4 ways-pin and a 2 ways-pin in the corresponding fixture, respectively. A clamp corresponds to an NC-block in the fixture. Furthermore, a clamped hole and a clamped slot correspond to a clamped 4wayspin and clamped 2ways-pin in that location, respectively. Section 2.1 clarifies the details about different types of locators and also pushers.

\subsection{Assembly fixture layout optimization}

The studies about the design and optimization of assembly fixtures can be divided into two categories. The first category includes studies that assume rigid parts and assembly, and optimize the locator positions. The second category encompasses the studies which take the compliant behavior of the assembly and springbacks into consideration. For compliant sheet metal assemblies, the parts can be over-constrained.

The studies in the first category, rigid assemblies, have mainly focused on multi-station assemblies in which the fixture layouts of all stations are optimized simultaneously. These studies employ a state-space modeling (SSM) that Jin et al. [6] developed in 1999. Kim and Diang [4] utilized this SSM of multi-station assemblies to optimize the location of locators in a $2 \mathrm{D}$ space. The objective of the optimization is to minimize the maximum sensitivity of the assembly to parts and fixture variations. Phoomboplab and Ceglarek [7] developed this method to optimize the location of locators in $3 \mathrm{D}$ geometries.

Cai et al. [8] presented a method to minimize the variation of a single sheet metal part in an N-2-1 locating scheme. They claimed that the variation comes only from the pin and slot, and therefore they optimized only the location of pin and slot in their method. Zhaoqing et al. [9] developed the previous modeling to consider the effects of slot orientation and optimize it. Xie et al. [10] and Tyagi et al. [11] have improved the optimization procedure by utilizing evolutionary optimization algorithms. Masoumi 
et al. [12] also utilized the non-linear SSM to optimize the fixture layout and the assembly sequence to minimize the variation of KPCs. They have mapped the $3 \mathrm{D}$ model to a plane to find the optimal locations in that plane.

Design and optimization of fixture layout for compliant assemblies was started by Camelio et al. [13]. They utilized the finite element method (FEM) to find the optimal position of locators when there is a deviation in a locator. Their objective was minimizing the final deviations of the assembly. Lia and Wang [14] presented the same procedure but they have considered the location of spot welds as a design parameter in addition to the location of locators. Das et al. [15] have developed a surrogate-based model to predict the variation of assemblies. They utilized the model to optimize $\mathrm{X}$ coordinates of locations of three locators so that the probability of having KPCs in a defined range is maximum. Franciosa et al. [16] presented a similar approach but with a different surrogate model and optimization algorithm.

Lu et al. [17] presented a method of optimizing locators for compliant sheet metal workpieces when an external load is applied to the part. Their work does not include an assembly procedure of sheet metals. Their study is conducted for a 4-2-1 locating scheme and they claimed that the first 6 locators can be optimized by considering the part rigid. Thus, only the last locator needs to be optimized by considering the deformations of the part when an external load is applied. Xing et al. [18] have utilized Method of Influence Coefficient (MIC) to predict the deformation of sheet metal parts in the presence of gravity. Then, they have optimized the location of locators so that deformations of the part are minimum. Yu et al. [19] have optimized the location of locators by generating a response surface model of the assembly model to predict the variations of the assembly. They have utilized the MIC method to simulate the assembly process and generate the response surface model. This method does not find the optimal number, type, and location of locators. It only fine-tunes the location of previously defined locators.

Table 1 presents a summary of previous studies on fixture layout optimizations for assemblies. The design parameters in studies that consider the assembly rigid are mostly holes and slot positions. When the parts are considered rigid, they cannot be fixated in more than six degrees of freedom. However, the sheet metal parts can often deform and be over-constrained in assembly fixtures. Moreover, in welded sheet metal assemblies, spring-back occurs after releasing the clamps. Accordingly, fixture layout optimization for compliant assemblies cannot be conducted when the assemblies are assumed to be rigid because the overconstrained assemblies cannot be simulated. Moreover, the effects of springback and the deformations due to external forces, including gravity, cannot be considered when the assembly is considered rigid.

The design parameters that are optimized in studies that consider the assembly as compliant are one or two coordinates of some clamp positions $(\mathrm{x}, \mathrm{y})$. Consequently, they have mapped the $3 \mathrm{D}$ geometry of the parts to a $2 \mathrm{D}$ plane $(x, y)$ or they have optimized the location of a point in one direction ( $\mathrm{x}$ or $\mathrm{y}$ ). These solutions can result in a great loss of the design space when the assembly has a complex $3 \mathrm{D}$ geometry. The type of locators, slot orientations, and the number and location of additional clamps are not optimized in these studies, to the best of our knowledge.

\subsection{Scope of the paper}

There are several design parameters in fixture layout optimization for compliant assemblies, including most of the assemblies in BIW, that are not addressed in previous studies. Consequently, there is a research gap in finding an optimal assembly fixture layout for this type of assemblies. This gap can be clarified as follows.

- Lack of a method to define the optimal number of supports (additional clamps that over-constrain each part).

- Lack of a method to define the optimal type and location of holes and slots in compliant assemblies.

- Lack of a method to define the optimal orientation of slots in compliant assemblies.

- Most of the presented methods for compliant assemblies are limited to assemblies in which the assembly can be mapped to a $2 \mathrm{D}$ surface or the design space is limited to fine-tuning the predefined area for each location.

This paper addresses these gaps by presenting a novel method of design and optimization of compliant assembly fixtures. The method will define the optimal type and location of holes, slots, and clamping units in compliant assembly fixtures. The presented method is not limited to any type of geometry and can be applied to complex 3D assemblies. This method can be applied to compliant multi-station assemblies in a top-down design procedure.

To attain this goal, compliant variation simulation tools are utilized along with Genetic Algorithms (GA). The method presented includes two stages. In the first stage, the type and a rough location of holes, slots (and slot orientations), and clamps will be determined among all feasible areas. Thereafter, in the second stage, these locations will be fine-tuned. Compliant sheet metal parts in BIW are mostly joined by spot welds. Therefore, this paper focuses on this type of joints. Nevertheless, the presented method can be utilized for other types of joints as well. 


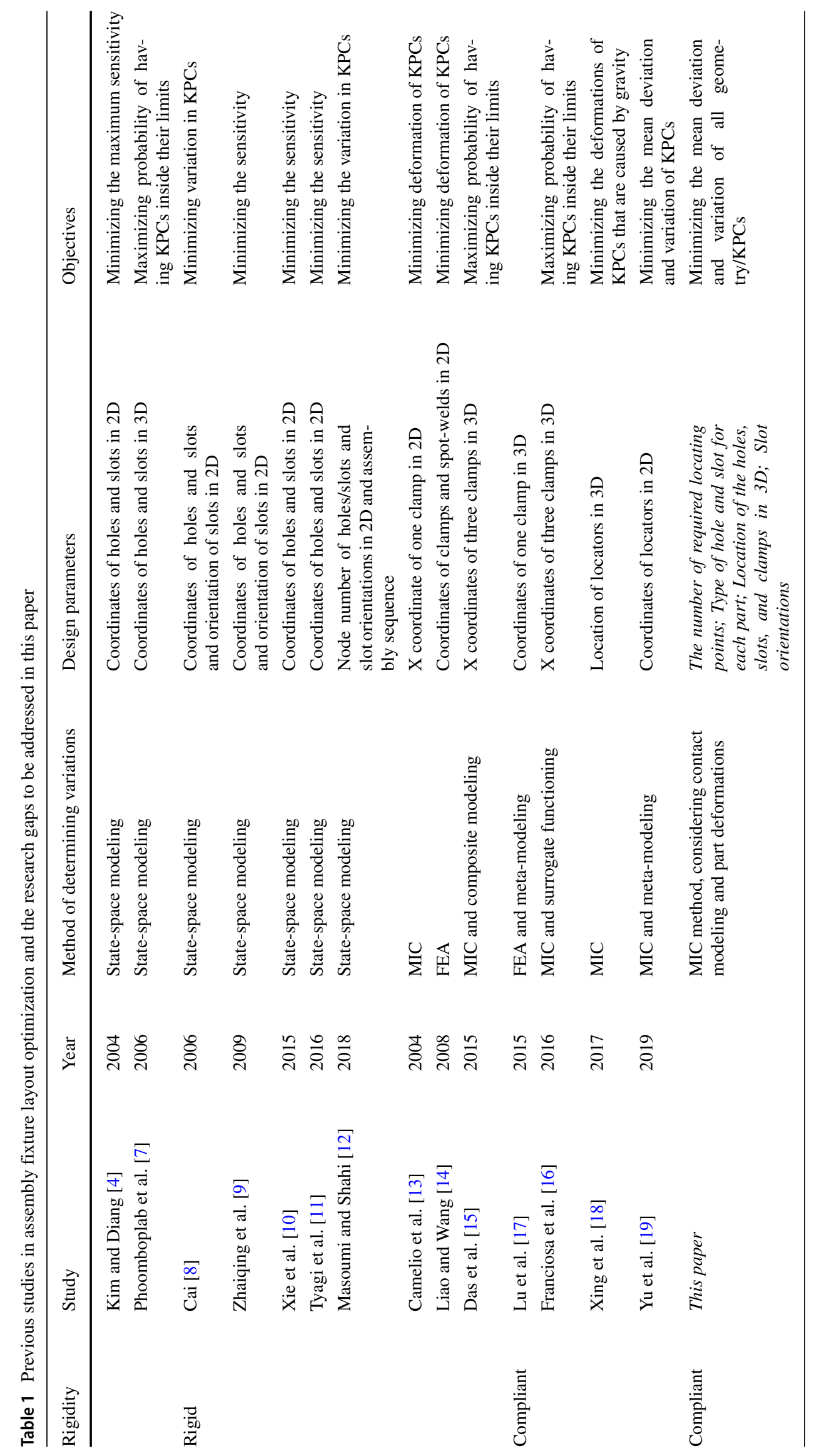


The problem of fixture layout design, including the assumptions, design parameters, objectives, and constraints is clarified in Section 2. Section 3 illustrates the utilized method of predicting the variations of compliant assemblies. The method of solving the problem is elaborated in Section 4. Section 5 presents two sample cases in which the method is applied to each case. Section 6 addresses the discussions and future works.

\section{Problem description}

A fixture layout optimization is a problem of finding the optimal number, positions, and types of locators for each part in the assembly. Section 2.1 clarifies the problem definition and assumptions made. The entire area of a part cannot be a candidate locator position due to practical limitations. Moreover, considering the continuous 3D geometries of the parts as the design parameters builds up to the complexity of the optimization. Section 2.2 addresses these concerns by converting the continuous areas of parts to discrete areas that are practically feasible to be a locator position. Thereafter, the design parameters, objective of optimization, and constraints are clarified in Sections $2.3,2.4$, and 2.5 , respectively.

\subsection{Problem definition and assumptions}

An assumption made in this paper is that each part has one hole and one slot. A hole always locks the movements in two perpendicular directions in the tangent plane to its locator position (two degrees of freedom). If the hole is clamped, it locks the movement in the normal direction to the tangent plane, in addition to the in-plane movements. A slot always locks the in-plane movement of the part in the perpendicular direction to the slot orientation (one degree of freedom, see Fig. 3). If the slot is clamped, it locks the transformation in the normal direction in addition to the perpendicular direction to the slot orientation. A clamp locks the movements in the normal direction to the tangent surface of its position (one degree of freedom).

Pushers are normally utilized when the goal is to support the part against a force in a certain direction, e.g., gravity force. Another application of pushers is when it is not practicable to use a complete clamping unit there or one side of the part is unaccessible. Pushers are considered the same as clamps in the simulations. This is because usually pushers are used when a part cannot or will not move on the free side of the pusher direction. The designer can, for each case, decide if a pusher should replace a clamp in the fixture. Table 2 lists different types of locators and their properties.

In practice, there are some special cases in which a part has more than one hole/slot or no hole/slot. However, this
Table 2 Different types of locators and their properties

\begin{tabular}{lll}
\hline Type & Locking DoF & Direction \\
\hline Clamp & 1 & Normal \\
Hole & 2 & In-plane \\
Slot & 1 & In-plane \\
Clamped hole & 3 & In-plane and normal \\
Clamped slot & 2 & In-plane and normal \\
\hline
\end{tabular}

paper addresses the cases in which each part has one hole and one slot because this is the most common case in the automotive industry. The following list presents a summary of all assumptions.

- Each part has one hole and one slot in which they can be clamped or not.

- A part can be over-constrained meaning that additional clamps can be utilized to lock more than six degrees of freedom of the part.

- Pushers and clamps are considered the same in simulations (normal direction is locked) and the designer can decide to replace the clamp if the part does not need to be locked in both sides.

\subsection{Discretization of the design space}

There are a variety of practical limitations that should be considered in a fixture layout design. For instance, sharp curves cannot be considered potential locator positions or some areas are not accessible in the fixture and cannot be candidates for locator positions. Consequently, these areas should be subtracted from the design space. Moreover, considering $\mathrm{x}, \mathrm{y}$, and $\mathrm{z}$ as continuous optimization parameters to find the position of locators requires defining some complex constraints to limit the values of these parameters into the areas of parts in the space. Furthermore, there is a limit of the minimum distance between two locators in a fixture.

This paper addresses these concerns by subtracting the areas that cannot be locator positions from the design space. Thereafter, the feasible areas are divided into smaller areas where a locator can be positioned. These areas are referred to as candidate locating areas. Each candidate locating area can be location of only one locator. Accordingly, the optimization problem will be to find areas among all candidate locating areas that should be selected as locating areas and also the type of locator (clamped hole, hole, clamped slot, slot or clamp) that should be utilized in each selected area.

The position of the locator in each area is referred to as locator position. The locator positions are considered roughly in the center of each candidate locating area. 
Subsequently, after defining the optimal areas and type of locators in them, the locator positions are fine-tuned in another optimization process.

The size of each candidate area should not be smaller than the minimum required distance between two locators. It should neither be so large that changing the locator position inside that area results in a significant difference in the robustness of the layout and geometrical quality of the assemblies. Consequently, the minimum and maximum size of areas are case dependent and should be defined based on the unique properties of each assembly.

Figure 1 displays the discretization of a simple example. The feasible areas of the design space are indicated in gray in this figure. Thereafter, these areas are divided into smaller areas to generate candidate locating areas. Accordingly, the design space is discretized into six candidate locating areas in this sample.

\subsection{Design parameters}

Based on the problem definition and the assumptions, a fixture layout can be defined by the following parameters: location of the hole and if it is clamped or not, location of the slot, the orientation of it and whether it is clamped or not, and the number of additional clamps and their locations. These parameters are represented by four variables that are listed in Table 3 and elaborated in this section.

Figure 2 depicts a simple example of utilizing these parameters in an assembly. The feasible area in each part of this assembly is divided into four and eight candidate areas for parts 1 and 2, respectively. Two indices, $p$ and $q_{p}$, are utilized to represent different parts and candidate areas in each part, respectively. Correspondingly, $P$ represents the total number of parts in the assembly, and $Q_{p}$ represents the total number of candidate areas in part $p$. Therefore, in the presented sample by Fig. 2, $P=2, Q_{1}=4$, and $Q_{2}=8$.

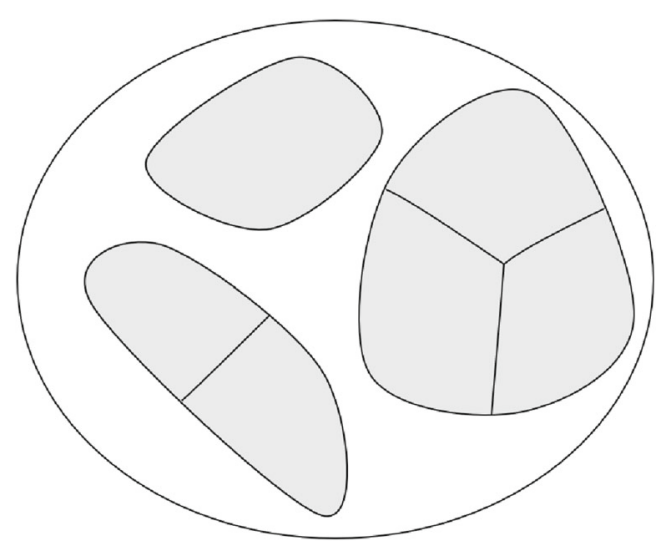

Fig. 1 A sample of discretization of the design space
Not all candidate locating areas can be hole/slot candidate areas. Having the hole and slot in planes that are more or less perpendicular to each other leads to a cumbersome locating of parts in the fixture. Therefore, it is preferred to have the hole and slot in roughly parallel planes. Hence, among all candidate locating areas, some of them may not be proper for hole/slot. Accordingly, the total number of candidate locating areas that can also be the location of the hole/slot is presented by $H_{p}\left(H_{p} \leq Q_{p}\right)$. The candidate area numbers $\left(q_{p}\right)$ are assigned to these areas firstly, and then to the rest of the candidate areas. Therefore, the candidate areas number 1 to $H_{p}$ represent the areas which are proper for hole/slot and clamps. The remaining candidates, from $H_{p}$ to $Q_{p}$, can only be the location of clamps. In the sample, presented in Fig. 2, it is supposed that all candidate areas of part 1 can be a hole/slot location, but candidate areas 7 and 8 in part 2 cannot be the location of a hole/slot. Therefore, $H_{1}$ and $H_{2}$ are 4 and 6 , respectively.

Two variables, $\beta_{1_{p}}$ and $\beta_{2_{p}}$, are introduced to represent the candidate area number in which the hole and slot are located in part $p$. In the presented sample, $\beta_{1_{1}}=1$ and $\beta_{2_{1}}=4$ indicate that the hole location for part 1 is candidate area 1 , and the slot location for this part is candidate area 4 . Similarly, $\beta_{1_{2}}=3$ and $\beta_{2_{2}}=2$ represent that the locations of the hole and slot of part 2 are candidate areas 3 and 2, respectively.

To represent the number and location of clamped areas for each part, $\alpha_{q_{p}}\left(q_{p}=1,2 \ldots, Q_{p}\right)$ is introduced. This parameter clarifies whether each candidate area $\left(q_{p}\right)$ is clamped or not. As a result, the value of $\alpha_{q_{p}}$ can only be 0 or 1 for each candidate area where 1 represents that the corresponding candidate area is clamped and 0 represents the opposite. The value of $\alpha_{q_{p}}$ for candidate areas $\left(q_{p}\right)$ that are assigned to the hole/slot defines whether the hole/slot is clamped or not. Accordingly, if $\alpha_{\beta_{1, p}}$ is equal to 1 , the hole in the part $p$ will be a clamped hole and if it is 0 it will be a hole which is not clamped. The same applies to slots. In the sample presented by Fig. 2, the candidate areas 2 and 4 in part 1, and the candidate areas 1, 3, and 6 in part 2 are clamped. Since candidate area 4 in part 1 is also slot location, the slot will be a clamped slot. The same applies to the hole in part 2.

Each slot locks the translation of its locating area in the perpendicular direction to the slot orientation and in the normal direction if it is a clamped slot. The normal direction is demonstrated by vertex $N$ in Fig. 3. To represent the locking direction of the slot in the in-plane direction, $\theta_{p}$ is introduced. This parameter indicates the angle between the in-plane locking direction of the slot and a fixed vector in the same plane.

The fixed vector is presented by $\mathbf{A}$. This vector should be orthogonal to the normal vector $\mathbf{N}$. Therefore, $\mathbf{A}$ can be 
Table 3 Design parameters

\begin{tabular}{lll}
\hline Parameter & Variable & Feasible values \\
\hline Being clamped or not for candidate area $q$ of part $p$ & $\alpha_{q_{p}}$ & 0,1 \\
Assigned candidate area to the hole in part $p$ & $\beta_{1, p}$ & $1,2, \ldots, H_{p}$. \\
Assigned candidate area to the slot in part $p$ & $\beta_{2, p}$ & $1,2, \ldots, H_{p}$. \\
In plane locking direction of slot in part $p$ & $\theta_{p}$ & {$[0180]$} \\
\hline
\end{tabular}

determined from the cross product of $\mathbf{N}$ and an arbitrary vector, $\mathbf{a}$, where $\mathbf{a}$ is not equal to $\mathbf{N}$, and is fixed. Equation 1 presents this relation.

$\mathbf{A}=\mathbf{N} \times \mathbf{a}$

Having $\mathbf{A}, \mathbf{N}$, and $\theta_{p}$, the in-plane locking direction of the slot can be determined. To utilize this direction in the simulation of the assembly procedure for locking it, it should be defined as a unit vector in the global coordinate system of the model $(x y z)$. Hence, it should be defined as a vector in the local coordinate system of the slot $(A B N)$ firstly, and then be transferred to $x y z$ coordinate system. The local coordinate system of each slot can be defined by three vectors of $\mathbf{A}, \mathbf{N}$, and $\mathbf{B}$. $\mathbf{A}$ and $\mathbf{N}$ are previously defined and $\mathbf{B}$ can be determined using Eq. 2.

$\mathbf{B}=\mathbf{N} \times \mathbf{A}$

Subsequently, Eq. 3 is used to determine the in-plane direction of the slot in its local coordinate systems.

$\mathbf{d}=\cos (\theta) i+\sin (\theta) j+0 k$

Having coordinates of the slot location in the global coordinate system, the transformation matrix $\mathbf{Q}$ can be defined. Accordingly, the unit vector of the in-plane locking direction in a global coordinate system can be determined using Eq. 4.

$\mathbf{d}^{\prime}=\mathbf{Q}^{-1} \mathbf{d}$

Utilizing the parameters presented in this section, the number of locators and their types (clamped hole, hole, clamped slot, slot, or clamped) and the area for each locator can be defined. Hence, utilizing an optimization algorithm to determine the optimum value of these parameters results in determining the optimal fixture layout of the assembly. Afterwards, to find the optimal position of each locator in the defined areas, the selected areas can be divided into smaller areas and the locating positions can be fine-tuned in that area. Accordingly, the optimization procedure will be carried out in two different stages. In the first stage, the optimal values of all design parameters will be obtained. Thereafter, in the second stage, the position of the defined locators in the first stage will be fine-tuned. Section 4 elaborates this procedure in detail.

\subsection{Objective function}

In mass production of assemblies, KPCs of each assembly can deviate from their nominal values. Therefore, the overall geometrical quality of assemblies can be assessed by the
Fig. 2 Illustration of the introduced designed parameters of a fixture layout in a simple example

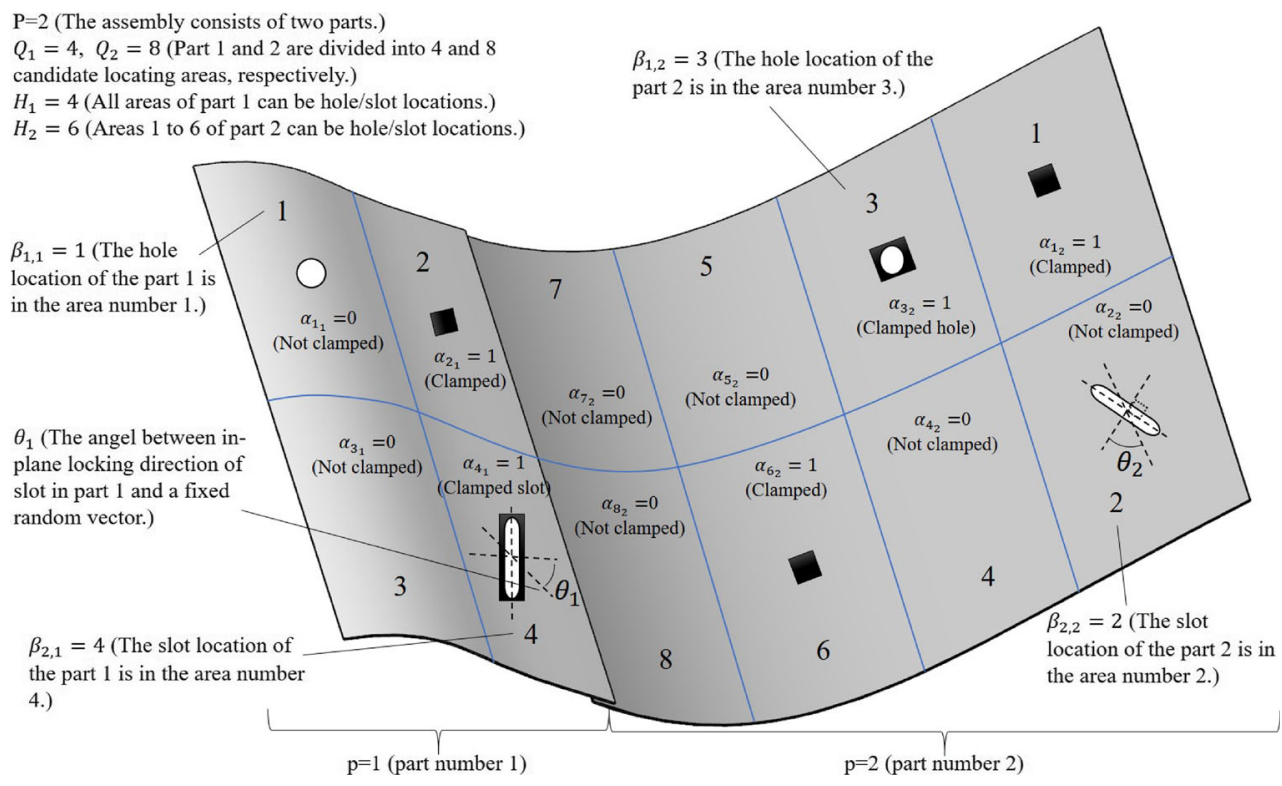




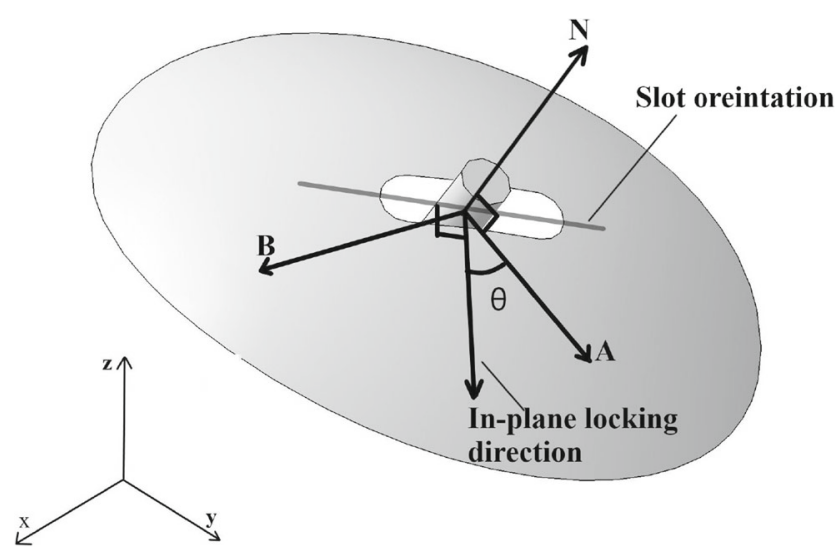

Fig. 3 Locking directions of a slot

mean deviation $(\mu)$ and variation, six times the standard deviation $(6 \sigma)$ of KPCs. Considering $d_{i j}$ as the magnitude of deviation of the $i^{\text {th }}$ KPC in the $j^{\text {th }}$ assembly, Eqs. 5 and 6 represent the definition of $\mu_{i}$ and $\sigma_{i}$, respectively. The deviations $\left(d_{i j}\right)$ after springback will be predicted by compliant variation simulations of the assembly. Monte Carlo simulations along with MIC method are utilized to conduct these simulations. The details of the utilized method are described in Section 3.

$\mu_{i}=\frac{1}{N} \sum_{j=1}^{N} d_{i j}$

$6 \sigma_{i}=6 \sqrt{\frac{1}{N-1} \sum_{j=1}^{N}\left(d_{i j}-\mu_{i}\right)^{2}}$

Here, $N$ is the number of iterations that are conducted to simulate the assembly process.

To consider the variation and the mean deviation of all KPCs, the root mean square (RMS) of $6 \sigma$ and $\mu$ of all KPCs are calculated as represented by Eqs. 7 and 8 . The variable $M$ in these equations indicates the number of all KPCs in the assembly.

$$
\begin{aligned}
& R M S_{v}=\sqrt{\frac{1}{M} \sum_{i=1}^{M}\left(6 \sigma_{i}\right)^{2}} \\
& R M S_{m}=\sqrt{\frac{1}{M} \sum_{i=1}^{M}\left(\mu_{i}\right)^{2}}
\end{aligned}
$$

A weighted sum of $R M S_{v}$ and $R M S_{m}$ can be considered as the objective of optimization as presented by Eq. 9 . The coefficient $\lambda$ is considered so $\lambda R M S_{m}$ becomes roughly the same as $R M S_{v}$. For the utilized sample cases in this paper, $\lambda=4$ is determined by conducting a number of variation simulations.

$$
f\left(\alpha, \beta_{1}, \beta_{2}, \theta\right)=R M S_{v}+\lambda R M S_{m}
$$

Changing the assembly fixture layout affects the geometrical quality of all points of the geometry and not only the KPCs. Therefore, in calculating the $R M S_{v}$ and $R M S_{m}$ deviation of the entire geometry can be taken into account, but with higher weights for KPCs. For the sample cases in this paper, the same weight is considered for the deviations of all nodes of the meshed geometries.

\subsection{Constraints}

The constraints of the problem based on the defined design parameters are clarified in this section. For each part, at least six degrees of freedom should be locked by the assembly fixture. This can be formulated as Eq. 10.

$2+1+\sum_{q_{p}=1}^{Q_{p}} \alpha_{q_{p}} \geq 6 \quad p=1,2, \ldots, P$.

The presented constraint by Eq. 10 is necessary to avoid rigid body motions of a part, but it is not adequate. To avoid rigid body motions of each part, the locating scheme of the part should be deterministic. Asada et al. [20] presented a Jacobian matrix of constraint equations or locating matrix and proved that if the rank of this matrix is equal to 6 , the locating scheme is deterministic. Based on this matrix, having location and direction of each locating point, the matrix $W$ can be formulated as demonstrated by Eq. 11 [21]. In this equation, the coordinates of the locating point $q_{p}$ are presented by $\left[x_{q}, y_{q}, z_{q}\right]$ and the normal vector of the direction in which this point is locking is presented by $\left[a_{q}\right.$, $b_{q}, c_{q}$ ]. Accordingly, if there is a clamped hole or clamped slot in a point, three or two rows, respectively, of the matrix will be considered for these points.

$W=\left[\begin{array}{llllll}a_{1} & b_{1} & c_{1} & c_{1} y_{1}-b_{1} z_{1} & a_{1} z_{1}-c_{1} x_{1} & b_{1} x_{1}-a_{1} y_{1} \\ \vdots & \vdots & \vdots & \vdots & \vdots & \vdots \\ a_{q} & b_{q} & c_{q} & c_{q} y_{q}-b_{q} z_{q} & a_{q} z_{q}-c_{q} x_{q} & b_{q} x_{q}-a_{q} y_{q} \\ \vdots & \vdots & \vdots & \vdots & \vdots & \vdots \\ a_{f} & b_{f} & c_{f} & c_{f} y_{f}-b_{f} z_{f} & a_{f} z_{f}-c_{f} x_{f} & b_{f} x_{f}-a_{f} y_{f}\end{array}\right]$

Here, $f$ indicates the number of all locating points when each hole is considered as three locating points and each slot is considered two locating points. Accordingly, if $W_{p}$ represents the locating matrix of the part $p$, the second constraint will be as represented by Eq. 12 .

$\operatorname{rank}\left(W_{p}\right) \geq 6 . \quad p=1,2, \ldots, P$

Another constraint of the problem is that there cannot be a hole and a slot in the same location simultaneously. This constraint is presented by Eq. 13 .

$\beta_{1, p} \neq \beta_{2, p}$ 
The rest of the constraints are the feasible values for each variable presented in Table 3.

\section{Compliant variation simulations}

The assembly procedure of spot-welded sheet metals can be described as the following steps [22].

1. The sheet metal parts are positioned into the assembly fixture.

2. The parts are clamped into their nominal positions.

3. The parts are joined (welded).

4. The clamps are released and springback occurs.

This process can be simulated using FEM to predict the final geometrical outcome of the assembly. To simulate one assembly procedure, two FE simulations are required. The first simulation is performed to determine the clamping forces. Defining $b$ and $a$ as representatives of the assembly state before and after welding, respectively, Eq. 14 presents the relation between clamping forces $\left(F_{b}\right)$ and deviation of parts $\left(D_{b}\right)$. Accordingly, $\left[K_{b}\right]$ is the stiffness matrix of the assembly before welding.

$\left[F_{b}\right]=\left[K_{b}\right]\left[D_{b}\right]$

The stiffness matrix of the welded assembly $\left[K_{a}\right]$ can be obtained by connecting the nodes in which welding occurs. The forces after welding are considered identical to the clamping forces by assuming that only clamping applies forces and the weld guns do not impose deformations to the parts.

$\left[F_{b}\right]=\left[F_{a}\right]$

Therefore, deviations of the assembly after releasing the clamps can be determined by Eq. 16 .

$\left[D_{a}\right]=\left[K_{a}\right]^{-1}\left[F_{a}\right]$

In order to predict the variation of assemblies in mass production, thousands of these simulations should be carried out using Monte Carlo simulations. Nevertheless, FEM simulations can be too time-consuming to be performed in each Monte Carlo iteration. To address this problem, Liu and $\mathrm{Hu}$ [22] presented the Method of Influence Coefficient (MIC) for variation simulations of compliant assemblies. In this method, only two FEM analyses are performed in the model and based on that, a linear relation is developed between $\left[D_{a}\right]$ and $\left[D_{b}\right]$. Equation 17 demonstrates this relation. The matrix $[S]$ in this equation is referred to as the sensitivity matrix.

$\left[D_{a}\right]=[S]\left[D_{b}\right]$

Several studies have built on this method to develop tools and improve their performance [23]. Camelio et al. [24] mixed this method with state-space modeling to predict variations in multi-station assemblies. Dahlström et al. [25] demonstrated that using the MIC method without considering contacts between the parts can cause crucial errors in the results. This is because parts can pass through each other if contact areas are not considered in the simulations. To avoid these errors, they developed a new method that combines MIC with contact modeling [25]. The relation between the deformations of parts and assembly is not linear when contacts are considered. Wärmefjord et al. [26] developed variation simulations using the MIC method along with contact modeling by automating the contact detections. The effects of weld sequence [27], heat [28], and non-linearity in materials [29] can also be considered in the simulations to improve the accuracy.

Compliant variation simulations can be conducted by several commercial programs, including $3 \mathrm{DCS}^{1}$ and $R D \& T^{2}$. Among these programs, RD\&T is capable of performing non-linear compliant variation simulations in which contacts between parts are considered by utilizing the method presented by Dahlström et al. [25]. In this method, the sensitivity matrix $[S]$ is dependent on the input variations because different deviations of mating parts might change the contact points and forces. Accordingly, this matrix is calculated for each input deviation separately. This method of calculation will considerably reduce the calculation costs compared with the direct method of determining deviations considering contacts between parts [25]. Gravity is another factor that is considered in this program and neglecting it can introduce errors to the simulations, particularly in assemblies where the sheet metal parts are relatively large and thin. Thus, this program is utilized in this study to predict the variation of assemblies after springback. Accordingly, in each objective function evaluation, this program conducts variation simulations of the model for the defined fixture layout by the optimization algorithm.

The geometrical quality of assemblies is a function of uncertainties (variations) that are associated with geometrical quality of parts, fixtures, tools, etc. Accordingly, in Monte Carlo simulations, these variations should be considered. The main sources of variations in sheet metal assemblies are part variations because usually fixtures are produced with relatively higher precision than parts. Several studies are focused on predicting the part variations based on the conditions in which the parts are produced [30,31]. Skin modeling is another method of considering variations in simulations [32].

In this study, the scanned data of the produced parts are utilized for simulations for the sample cases presented

\footnotetext{
$\overline{1_{\text {www.3dcs.com }}}$

${ }^{2}$ www.rdnt.se
} 
in Section 5. Hence, fifty deformed parts are modeled for each part and a random combination of them is utilized in each iteration of the simulation. Subsequently, to predict the geometrical quality of a specific fixture layout, thousand iterations of simulations are performed.

\section{Optimization method}

The optimization problem, as is defined in Section 2, can be represented as follows.

$\min f\left(\alpha, \beta_{1}, \beta_{2}, \theta\right)=R M S_{v}+\lambda R M S_{m}$

Subject to:

$\alpha_{q_{p}} \in\{0,1\}$

$\beta_{1, p}, \beta_{2, p} \in\left\{1,2, \ldots, H_{p}\right\}$

$\beta_{1, p} \neq \beta_{2, p}$

$0 \leq \theta_{p}<180^{\circ}$

$\sum_{q_{p}=1}^{Q_{p}} \alpha_{q_{p}} \geq 3$

$\operatorname{rank}\left(W_{p}\right) \geq 6$.

The objective function of this optimization problem is a complex non-linear function that requires running thousand iterations of simulations of a non-linear variation simulation model. Accordingly, the objective function is too complex to be solved by deterministic optimization methods. Therefore, meta-heuristic optimization methods are sound optimization methods to solve this problem.

Genetic Algorithms (GA) are the foremost meta-heuristic optimization methods that are relatively better recognized and developed. This paper develops a GA to solve the optimization problem presented.

The developed GA to solve the optimization problem is elaborated in this section. An introduction to GAs and the overall optimization procedure is presented in Section 4.1. Section 4.2 illustrates the mapping which is utilized to transfer the fixture layout properties (phenotype) to a solution in the GA (genotype). The utilized method to select the solutions for generating new solutions is illustrated in Section 4.3. Sections 4.4 and 4.5 present the utilized crossover and mutation procedures, respectively. The finetuning stage is explained in Section 4.7. Section 4.8 presents the procedure of utilizing the presented method in design of fixture layouts for compliant multi-station assemblies.

\subsection{Genetic algorithms}

Genetic Algorithms are from the class of evolutionary algorithms. To start the optimization process, these algorithms generate some random solutions (chromosomes) to the problem (initial population). Then, the value of the objective is evaluated for each solution by the objective function (function evaluation) and a proportional fitness is allocated to each solution. The solutions with greater fitness values receive a higher chance to produce new solutions (children) through crossover and mutation operations. Subsequently, the new solutions which have greater fitness values replace the solutions with lower fitness values. This procedure (optimization iteration) will continue until the convergence criteria are fulfilled. Hence, the best solution will be introduced as a minimum. A major drawback of the meta-heuristic optimization methods, including GAs, is that there is no guarantee that the final solution will be the global optimum of the problem. Nevertheless, a solution that is good enough for the problem can usually be found.

\subsection{Phenotype-genotype mapping}

The design domain of the fixture layout (phenotype) should be encoded to GA solutions (genotype). Accordingly, each solution in GA should represent a fixture layout for the assembly procedure. In other words, the values of all design parameters of $\alpha_{q_{p}}, \beta_{1, p}, \beta_{2, p}$, and $\theta_{p}$ for all $q_{p}$ and $p$ should be defined in each solution. To achieve the greatest convergence rate in the optimization, the mapping between phenotype and genotype should be one to one, i.e., for each solution in phenotype there should only be one and one solution in genotype [33].

Each fixture layout should have a set of all design parameters for each part of the assembly. Therefore, each solution of the GA should have a subset (sub-string) for each part. Accordingly, the number of sub-strings in each solution is equivalent to $p$. Each cell of every sub-string represents the value of a design parameter and is referred to as a gene. Therefore, the length of each sub-string will be $3+Q_{p}$ genes.

Figure 4 displays a sample solution (chromosome) of a fixture layout in GA. The corresponding assembly consists of two parts in which there are seven $\left(P_{1}=7\right)$ and nine $\left(P_{2}=9\right)$ candidate areas in the first and second parts, respectively. It is supposed that all candidate areas can be a hole/slot location $\left(H_{p}=Q_{p}\right)$.

In this sample layout, the location of the hole in the first part is in the fifth candidate locating area: $\beta_{1,1}=5$ and the hole is not clamped because the value of $\alpha_{5_{1}}$ is 0 . Subsequently, the location of the slot in the first part is the second candidate locating area, $\beta_{2,1}=2$, and the value of $\alpha_{2_{1}}$ is 1 . Therefore, there will be a clamped slot in the second candidate area of the first part. The slot locks two directions. The first direction is along the normal vector of the target place. The second direction is defined by the third string of the solution, and it is $38^{\circ}$ from the random vector of $\mathbf{A}$ in the normal plane of the position. 
Fig. 4 A sample solution (chromosome) in the GA for a fixture layout of assembly of two parts in which the first part has 7 candidate positions and the second part has 9 candidate positions

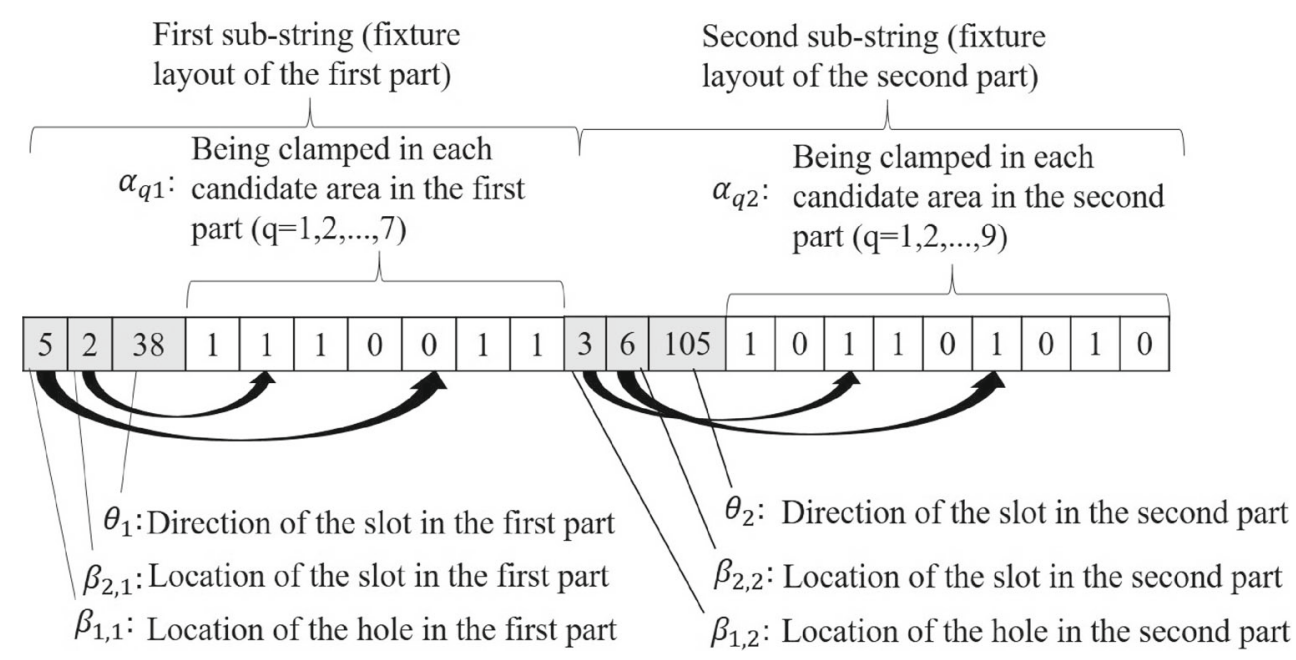

There are six locator positions in the first part out of all seven candidate areas. They are the first, second, third, sixth, and seventh candidate areas. Among them, the fifth and the second locating areas are a hole and a clamped slot, respectively, and the rest are clamps that lock the normal direction of the position in which they are located. Hence, this solution presents a 5-2-1 locating scheme for the first part.

There are six locator positions for the second part out of nine candidate positions. This part has a clamped hole in the third candidate locating area and a clamped slot with $105^{\circ}$ in the sixth candidate locating area. There are clamps in the first, fourth, and eighth candidate positions. Correspondingly, the presented locating scheme for the second part is also a 5-2-1 locating scheme.

\subsection{Selection}

To perform crossover operation, the parents should be selected among all solutions of the population. This study utilizes the Roulette Wheel method (see Ref. [34]) for this aim. Therefore, a fitness value should be allocated to each solution so that solutions with a lower value of the objective receive a higher fitness value. Therefore, Eq. 19 is developed as the fitness function.

fitness $(x)=\frac{1}{f(x)}=\frac{1}{R M S_{v}+\lambda R M S_{m}}$

Having the fitness value of each solution, a proportional probability is associated with the solution to be selected for crossover operation. Equation 20 defines the relation between probability of selection of the $i^{\text {th }}$ solution and its fitness value (fitness $(i)$ ). In this equation, $N$ is the population size.

$p(i)=\frac{\operatorname{fitness}(i)}{\sum_{j=1}^{N} \operatorname{fitness}(j)}$

\subsection{Crossover}

The selected solutions are utilized in pairs (parents) to generate new solutions (children). The design parameters can be divided into two types based on the type of values they can take. The first type includes parameters that can take integer values and the second type can take real values. Accordingly, $\beta_{1, p} \beta_{2, p}$ and $\alpha_{q_{p}}$ are type 1 and $\theta_{p}$ is type 2 .

The crossover methods for real coded genetic algorithms are different from those for integer values. Therefore, the crossover is conducted in two phases. In the first phase, a one-point crossover is performed for integer values of the parents. In this type of crossover, the parents are divided into two sections, from a random position. Afterward, the children are generated by swapping the second sections of the parents.

In the second phase, $\theta_{p}$ s for children are generated by utilizing an arithmetic combination of $\theta_{p} \mathrm{~s}$ of the parents [35] as presented by Eqs. 21 and 22. The relation between the real values of children $\left(\theta_{\text {child } 1}\right.$ and $\left.\theta_{\text {child } 2}\right)$ with real values in the parents $\left(\theta_{\text {parent } 1}\right.$ and $\left.\theta_{\text {parent } 2}\right)$ is demonstrated as follows. The variable $\zeta$ represents a random number between 0 and 1 .

$\theta_{\text {child } 1}=\theta_{\text {parent } 1} \zeta+\theta_{\text {parent } 2}(1-\zeta)$

$\theta_{\text {child } 2}=\theta_{\text {parent } 2} \zeta+\theta_{\text {parent } 1}(1-\zeta)$

\subsection{Mutation}

The mutation operations are different based on the type of values that each parameter can take. Therefore, a random gene from the candidate solution for mutation is selected, firstly. Then, depending on the parameter the selected gene represents, the mutation operation is defined.

If the selected gene for mutation is either $\beta_{1, p}$ or $\beta_{2, p}$, its value will change to a random integer from 1 to $q_{p}$, so that 
$\beta_{1, p} \neq \beta_{2, p}$. If the selected gene is a $\alpha_{q_{p}}$, a binary mutation will be applied to it. In this type of mutation, the value of gene changes to 0 if its original value is 1 and vice versa. The mutation for $\theta_{p}$ is conducted by changing the existing value to a new random real value from 0 to 180 .

\subsection{Handling the constraints}

The utilized method to handle all introduced constraints in Section 2.5 is explained in this section. The coding introduced in Section 4.2 handles all constraints except three of them that should be handled separately. These constraints are the constraints related to the rigid body motions (Eqs. 10 and 12).

Fixture layouts that do not constrict the rigid body motions result in singular FE models in variation simulations. Consequently, the values of objectives cannot be obtained for the solutions which do not satisfy the aforementioned constraints. To avoid these solutions, the constraints are checked for each individual before objective function evaluation. If a solution does not satisfy the constraints, a relatively large value is assigned to its objective value (penalty) instead of running the simulations for it. The penalty value is considered as 1000 in this study. This is because the value of objective functions $\left(R M S_{v}+\lambda R M S_{m}\right)$ in the presented sample case in this study fluctuates between 0.5 to 4 by changing their fixture layouts. Accordingly, if a fixture layout does not satisfy Eqs. 10 and 12, the value of its objective function will be 1000 which is significantly larger than normal values of the objective function for other fixture layouts.

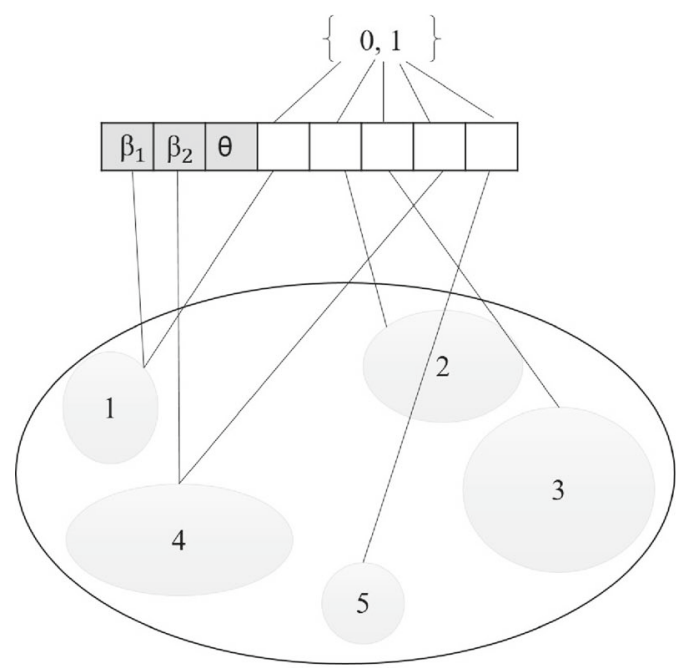

First stage: Optimal type (clamped/unclamped hole/slot) and location of locators, direction of the slot.

\subsection{Fine-tuning stage}

In the first stage of optimization, one node was considered from each candidate locating area as the representative of that area to find the optimal areas for locator positions. In this stage of optimization, the positions of the previously defined locating points within their locating areas are finetuned. Figure 5 demonstrates a schematic diagram of design parameters in each stage and their corresponding genotypes.

To fine-tune the locator positions, the surrounding area of each locator position of every locating area is divided into several new areas. Thereafter, a GA is utilized to find out how the optimal fixture layout from the first stage can be further improved by moving the defined locator positions. A chromosome in this GA has a gene for every locating area of each part. If a locating area is divided into $F$ smaller new areas, the gene corresponding to that area can take integers from 1 to $F$.

One point crossover operation is applied in this stage of optimization. The mutation is also similar to the first-stage optimization for integer values, in which a random integer will replace the selected gene for mutation.

Figure 6 illustrates the overall procedure of the method presented to find the optimal fixture layout of compliant assemblies.

\subsection{Multi-station assemblies}

Most of the assemblies in a BIW are produced in multiple stations [4]. Consequently, the datum positions of the assemblies in each station should be the same as the locating

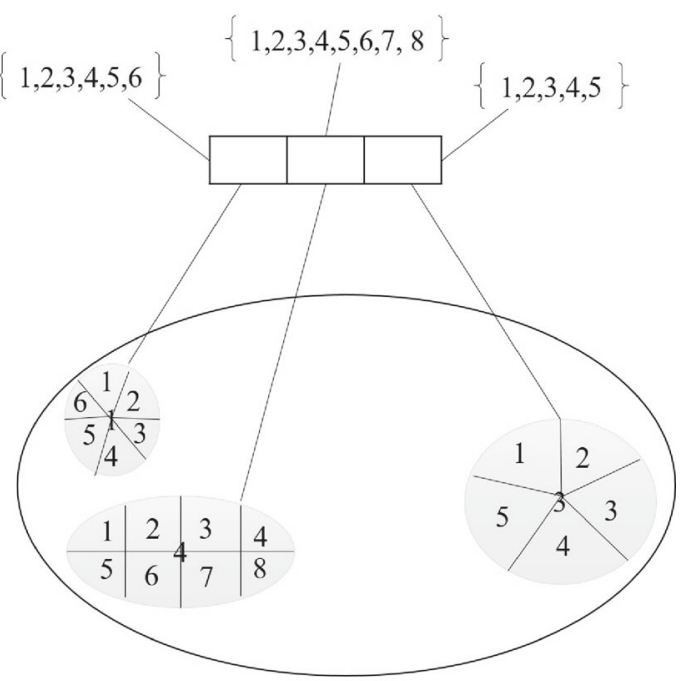

Second stage (Fine-tuning): To further improve the fixture layout by fune-tuning the locations.

Fig. 5 Phenotypes and genotypes in each stage of the optimization 


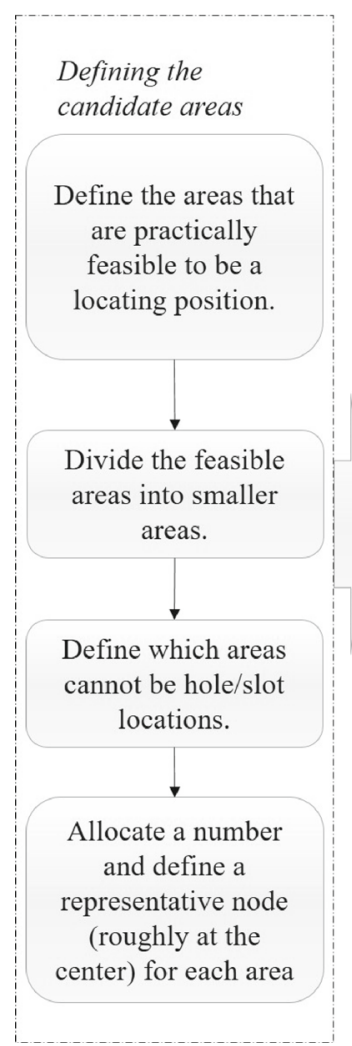

\section{First stage: Main stage}

Inputs: Candidate locating areas

Outputs: $\quad$ Locating areas, type of each locator (clamped

hole/clamped slot/hole/slot/clamp), directions of slots.

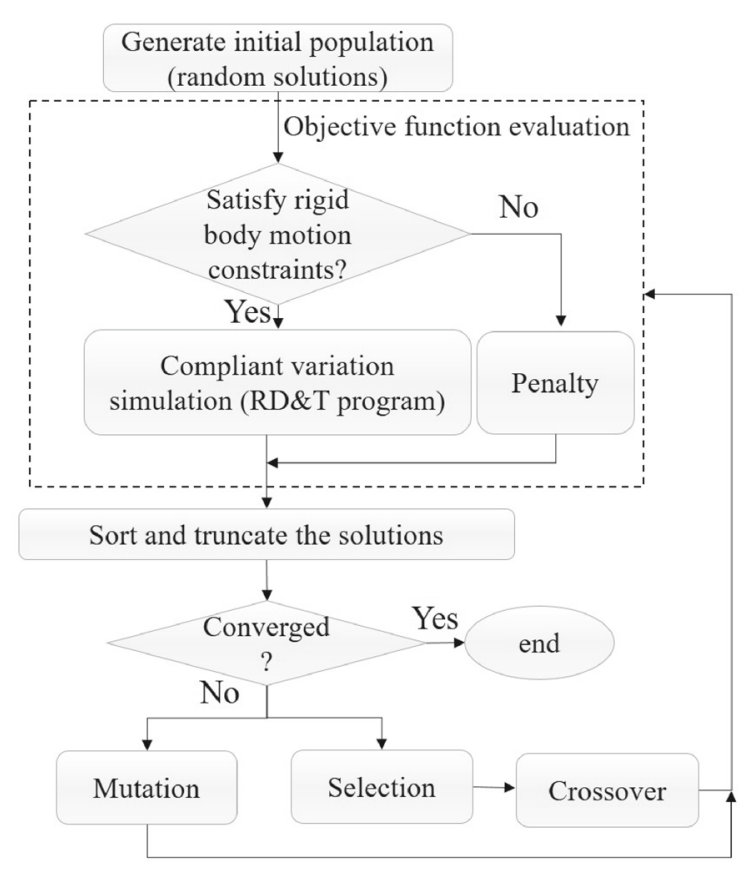

Second stage: Fine-tuning

Inputs: Optimal Fixture layout,

Representative neighbor nodes in each locating area

Outputs: Fine-tuned positions of the optimal fixture layout in each locating area

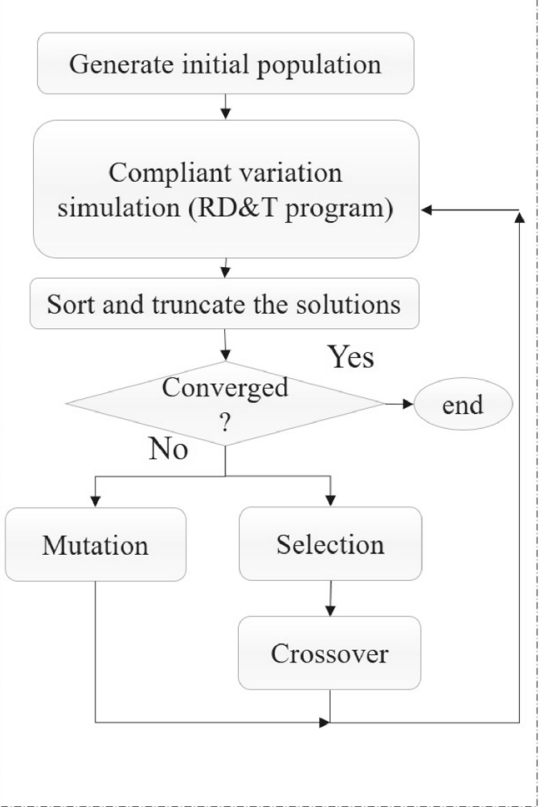

Fig. 6 The overall procedure of the fixture layout optimization presented in this paper

scheme of that assembly in the next station. Besides, the locating positions in each station should be reused in the the previous stations to avoid variation propagations [5]. Accordingly, the fixture layout of each station cannot be designed independently. This issue is addressed in the methods that utilize state-space modeling by optimizing the layouts of all stations simultaneously [4, 8-10, 12].

Optimizing the fixture layouts of all stations simultaneously when the compliant behavior is considered requires simulating variations of all stations in each function evaluation. Furthermore, the number of design parameters is significantly larger for compliant assemblies compared to rigid assemblies. Consequently, the optimization of all fixture layouts simultaneously is not applicable due to huge calculation cost and complexity, particularly when the number of stations is relatively large.

To overcome this issue, this paper proposes a top-down design procedure for designing fixture layouts for compliant multi-station assemblies.

The datum positions of the final assembly depend on its function and they are usually defined in positions wherein the assembly is going to be installed. The ultimate goal is to minimize the effects of variations so that the highest geometrical quality is achieved in the final station. In each station, the fixture layout of the next station for the input of the current station should be reused. Accordingly, these locations are considered as a constraint in fixture layout optimization of each station. Hence, their corresponding design parameters will be removed from the optimization. Therefore, having the datum positions of the last station, the optimal fixture layout of the last stations can be defined. The locating scheme of each sub-assembly in that fixture layout is the datum position of that assembly in the previous station. Based on those datum positions, the optimal fixture layouts of the previous station can be designed. This procedure continues until the fixture layout in the first station is defined.

Figure 7 illustrates the overall scheme of this procedure. In this schematic diagram, two assemblies from stations $i$ and $j$ are assembled in station $i+j$. The next station after $i+j$ is station $i+j+k$. Therefore, the datum positions of assembly $i+j$ are defined based on locating scheme of assembly $i+j$ in the assembly fixture of $i+j+k$. Subsequently, the optimal fixture layout of $i+j$ is designed adding a constraint that datum positions should be reused.

The reason for conducting simultaneous optimization of all fixture layouts in space-state modeling-based methods is that the locator positions that are defined in the subsequent stations and reused in the preceding stations are only optimal for the subsequent station and may not be optimal in the preceding stations [4]. However, the fixture layouts of the subsequent stations play a more important role in the 


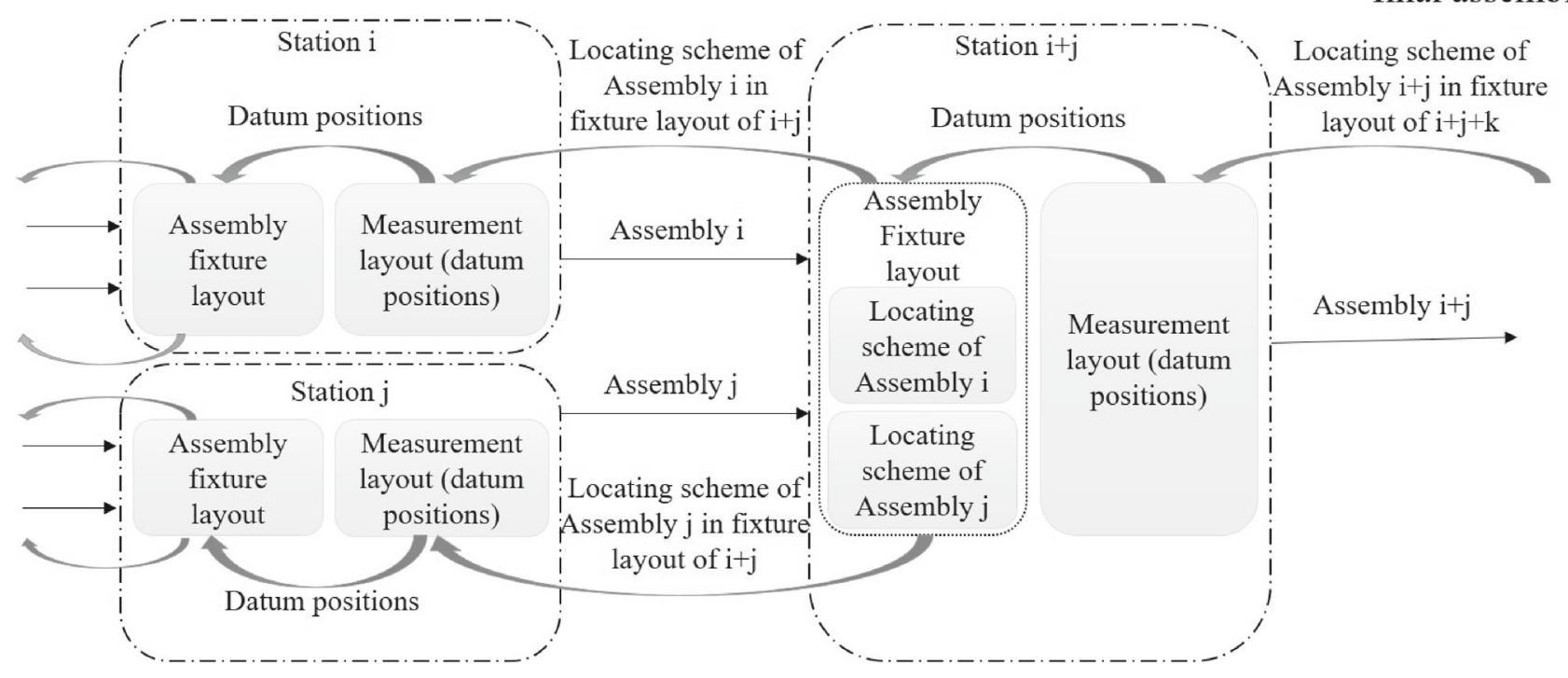

Fig. 7 The overall procedure of fixture layout optimization of compliant multi-station assemblies

variations of the final assembly compared with the fixture layouts of preceding stations. This is because even if the sub-assemblies of the preceding stations have a very low geometrical quality, but the fixture layouts of the subsequent stations have low robustness, they can amplify the effects of variations of the incoming sub-assemblies in the final assembly. On the other hand, if the fixture layouts of the subsequent stations have relatively higher robustness, they can reduce the effects of variations of the previous stations. Therefore, it is logical to start defining the optimal fixture layouts from the last stations and reuse their locator positions as a fragment of the layouts in the previous stations.

Moreover, normally, the locators that are repeated from the next stations are only a small fraction of all locators in each layout. As a result, the contribution of the repeated locators that are defined in the subsequent stations is not significant in the preceding stations. This hypothesis is proved for one of the presented sample cases in Section 5.

\section{Results}

The presented method is implemented by a MATLAB code with an interactive connection to RD\&T program for function evaluations. Thereafter, it is applied to two different sample cases. The second sample case is introduced because it has a special condition that requires some modification in the presented algorithm, to make it applicable for that sample case.
Both sample cases are taken from the automotive industry and there are already fixture layouts utilized to produce them. Therefore, the geometrical qualities of each assembly $\left(R M S_{v}\right.$ and $\left.R M S_{m}\right)$ for the fixture layout determined by the algorithm presented in this paper are compared with the geometrical qualities when the existing fixture layout is employed.

The same parameter settings are defined in all GAs that are utilized to solve the sample cases. The population size is set to 200 . The crossover and mutation rates are defined as 0.7 and 0.3 , respectively. Based on the type of operations and the number of variables, the defined settings lie within the range of optimal settings of the GAs [36]. The convergence criterion is defined as the maximum number of iterations which is set to 100 .

\subsection{Sample case 1}

The first sample case is a spot-welded assembly of three parts from the automotive industry. The geometry of this assembly is so that mapping it into any $2 \mathrm{D}$ plane will cause a significant loss of the design area. Hence, it will be very challenging to use the methods that utilize mapping geometry coordinates to a $2 \mathrm{D}$ plane to find an optimum fixture layout for this assembly. Figure 8 represents the datum positions of this assembly (the locating scheme of this assembly in the next assembly station).

The first step to find the optimal fixture layout for this assembly is to define the feasible areas. The second step is to divide these areas into smaller areas depending on the 


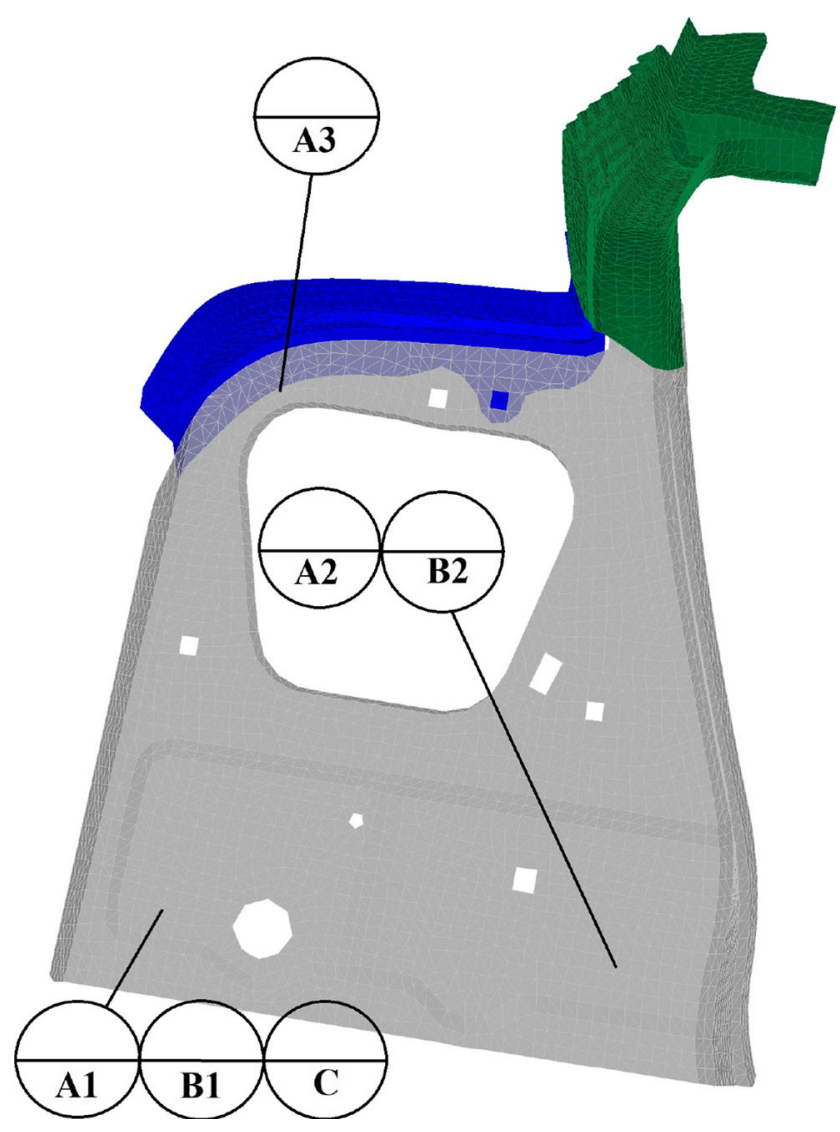

Fig. 8 Datum positions of sample case 1

size of the assembly and the required resolution in the first stage. The results of these steps for the first sample case are indicated in Fig. 9. The white spheres in this figure represent the spot welds.

The feasible areas of Filler Piece are divided into eight candidate positions $\left(Q_{1}=8\right)$. The number of candidate positions for Mounting plate and Water Channel are 15 $\left(Q_{2}=15\right)$ and $14\left(Q_{3}=14\right)$, respectively. Among candidate areas of Mounting Plate, candidate area numbers 12 to 15 cannot be hole/slot locations $\left(H_{2}=11\right)$. It is supposed that for the other two parts all candidate areas can be hole/slot locations $\left(H_{1}=Q_{1}, H_{3}=Q_{3}\right)$.

The candidate locating area numbers 1,3 , and 11 in Mounting Plate are the location of a clamped hole, a clamped slot, and a clamp in the next assembly station, respectively. Therefore, to reuse the locating scheme of the assembly of the next station in this fixture layout, $\beta_{2,1}, \beta_{2,2}, \theta_{2}$, and $\alpha_{11_{2}}$ are removed from the design parameters and the fixed hole, slot, and clamp are considered in candidate positions 1,3 , and 11 , respectively.
The optimization constraints are also adopted based on these conditions. Nevertheless, to assess the effect of applying these constraints, the optimal fixture layout is also obtained without limiting the hole and slot locations of Mounting Plate.

To evaluate $R M S_{v}$ and $R M S_{m}, 50$ deformed parts from each part are generated based on the scanned data of the produced parts. Therefore, for each function evaluation, 1000 variation simulation iterations are conducted among the deformed parts.

The optimal solution in the GA when the hole and slot locations of Mounting Plate are predefined is obtained as follows. The first, the second, and the third sub-strings represent the fixture layouts of the Filler Piece, Mounting Plate, and Water Channel, respectively.

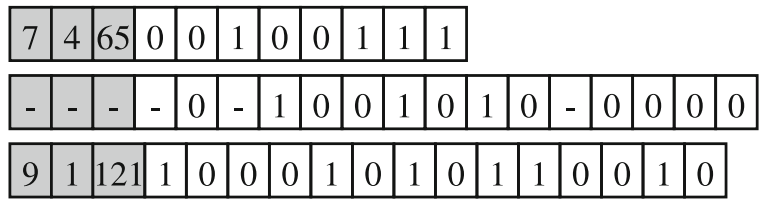

Figure $10 \mathrm{a}, \mathrm{b}$, and $\mathrm{c}$ illustrate the existing and the optimal fixture layouts without and with limiting locations of some locators to datum positions, respectively. The variation of each node is indicated by color coding in these figures. The gravity direction is also presented in the figures.

\subsection{Fine-tuning sample case 1}

The optimal layout that is obtained by restricting locations of some locators in Mounting Plate is fine-tuned to further improve the robustness of the fixture. The reason for finetuning is that the optimal fixture layout presents the optimal areas where locators should be located. However, it does not indicate the exact location of each locator in those areas. Hence, the optimal location of each locator in the selected areas can be defined in this stage.

The locations of the hole, the slot, and the clamp in Mounting plate are exempted from fine-tuning because they are considered exactly where the locators in the next assembly station are positioned. Therefore, there are 13 locating points in the optimal layout left for finetuning. Depending on the size of the area in each locator position, several neighbor nodes are chosen as candidates of fine-tuning. Table 4 represents the number of neighbor nodes that are chosen as candidate nodes to replace the locator positions. The first row of this table represents the locating areas based on the numberings in Fig. 9. The second row indicates the total number of neighbor nodes for the corresponding locating area, including the 


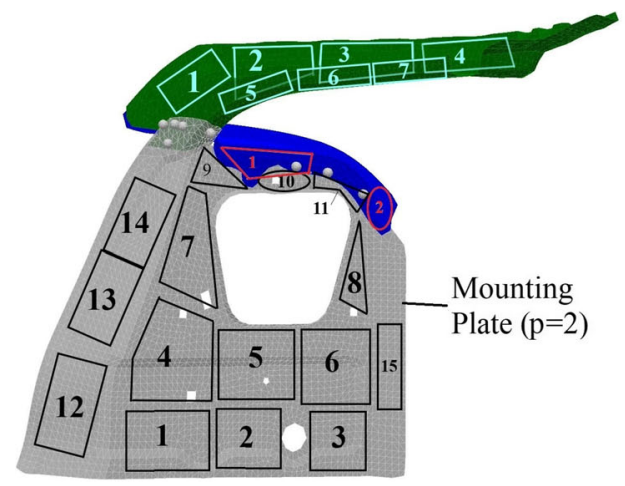

(a) Front view

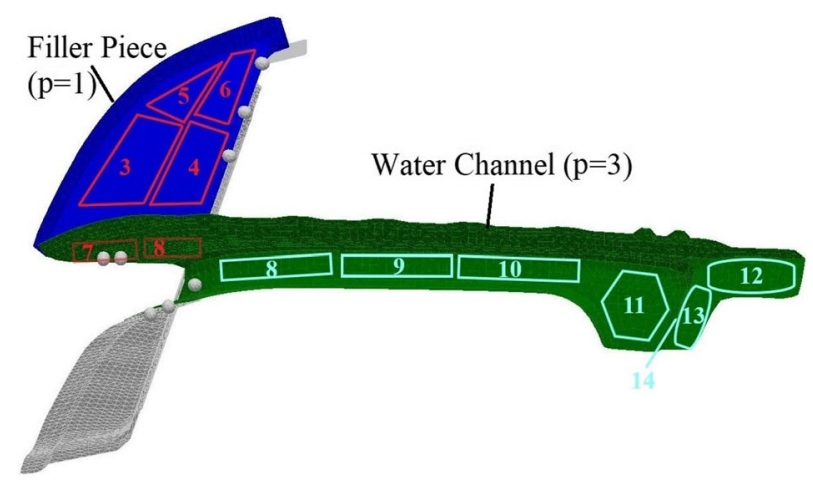

(b) Top view

Fig. 9 Candidate locating areas of each part in sample case 1

representative nodes which were utilized in the first stage of the optimization.

The optimal neighbor nodes to replace the previous representative of each area are obtained by running the presented GA in Section 4.7. The optimal solution in GA is obtained as follows.

$$
\begin{array}{|l|l|l|l|l|l|l|l|l|l|l|l|l|l|}
\hline 1 & 1 & 1 & 5 & 1 & 2 & 1 & 3 & 1 & 4 & 1 & 2 & 4 & 7 \\
\hline
\end{array}
$$

Each integer in this solution indicates which neighbor node is the optimal representative of its corresponding locating area. For example, in the fourth locating area in Filler Piece, candidate number 7 in Fig. 9 (shown in italics in Table 4 and the solution), the node neighbor number 5 should be replaced by the previous representative node of the area. The preliminary representatives of each area are indicated by number 1 in this solution.

Table 5 lists $R M S_{v}$ and $R M S_{m}$ of sample case 1 for the existing and the optimal layouts. The variations and the mean deviations are improved by $57 \%$ and $53 \%$ in the optimal layout when location of hole and slot are restricted to be the same as the next station. These criteria are further improved by $8 \%$ and $5 \%$ in the fine-tuning stage. The improvement is more or less the same when the locations of hole and slots in Mounting Plate are not limited to any place.

\subsection{Sample case 2}

The second sample case is an assembly of an automotive door panel. This assembly consists of two parts in which a ring will be assembled on a door panel. Figure 11 depicts the model of this assembly and its datum positions.

An additional constraint for sample case 2 is that the fixture layout of each part is not independent of the other part because the Ring is assembled on the Panel so that any locator in Ring will also locate the Panel. To address this constraint, the same candidate locating areas are chosen for

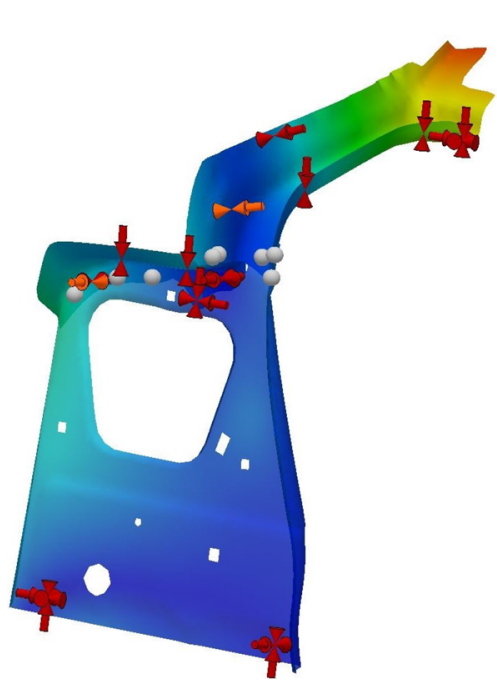

(a) Existing fixture layout.

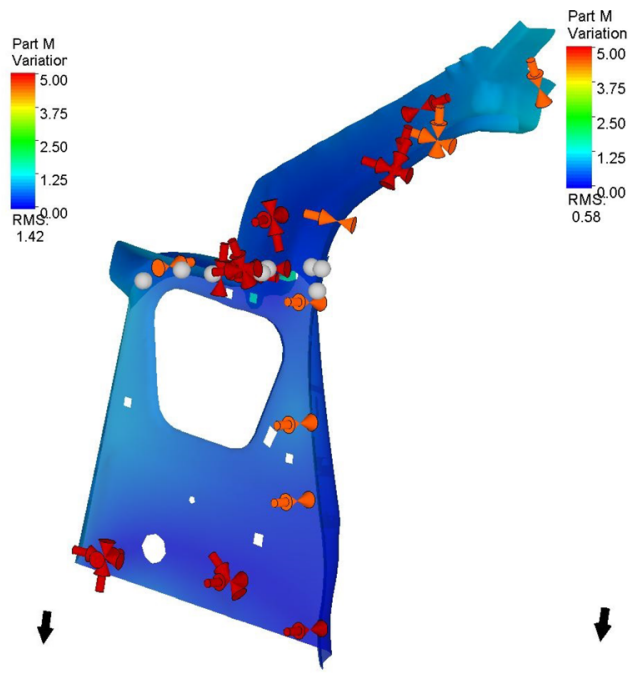

(b) Optimal fixture layout without limiting some(c) Optimal fixture layout when some locator lolocator locations to datum positions. cations are limited to datum positions.

Fig. 10 Existing and optimal fixture layouts and color coding of their variations in 1000 Monte Carlo iterations for sample case 1 
Table 4 The number of neighbor nodes for fine-tuning in each locating area in sample case 1

\begin{tabular}{llll}
\hline & Filler piece & Mounting plate & Water channel \\
\hline Area & 34678 & 1347911 & 15791013 \\
Neighbors & 53885 & $--677-$ & 747747 \\
\hline
\end{tabular}

both Ring and Panel wherein the Panel is covered by the Ring. Accordingly, the parameter $\alpha_{q_{p}}$ represents candidate positions of both parts when $q$ represents the common areas between the two parts. Consequently, when $q$ is representing a candidate position not covered by Ring, $\alpha_{q_{p}}$ represents only the Panel.

Figure 12 displays the defined candidate locator positions for the second sample case. There are 16 candidate positions for the Ring $\left(Q_{1}=16\right)$ and 28 candidate positions for the Panel $\left(Q_{2}=28\right)$ in which the first 16 positions are common with the candidate locating areas of the Ring. Accordingly, the design parameter $\alpha_{q_{p}}$ is restricted as follows:

$\alpha_{q_{1}}=\alpha_{q_{2}} \quad q=1,2, \ldots, 6$.

To apply the additional constraints of the second sample case in the GA, only one sub-string is considered in each solution. The first 16 genes of the solutions are common between both parts and the rest are only for the Panel.

Locations of the hole and the slot in the datum positions are in the common area between the two parts. Therefore, each part has already a hole and slot in the same position as datum positions and only the number and location of additional clamps can be optimized. Consequently, the design parameters regarding the hole and slot for both parts $(\beta)$ are removed from the optimization.

The geometrical qualities of the sample case 2 for each fixture layout are determined by conducting 1000 simulation iterations.

The length of each chromosome in the optimization of the second sample case is 25 (the corresponding genes to candidate areas 1, 3, and 27 are already used for datum positions) in which each gene can take only 0 and 1. Figure 13 depicts the original and optimal fixture layouts of the second sample case with the color-coding of their variations. The corresponding solution in GA is:

\begin{tabular}{|l|l|l|l|l|l|l|l|l|l|l|l|l|l|l|l|l|l|}
\hline- & - & - & - & 0 & - & 0 & 1 & 0 & 0 & 0 & 0 & 0 & 0 & 0 & 1 & 0 & 0 \\
\hline \hline 0 & 1 & 0 & 0 & 1 & 0 & 0 & 0 & 0 & 1 & 0 & - & 0 & & & & \\
\hline
\end{tabular}

\subsection{Fine-tuning sample case 2}

The optimal fixture layout of the second sample case has five locating areas. These areas are, based on the numbering in Fig. 12, 5, 13, 17, 20, and 25. The numbers of neighbor nodes considered for each area are 7, 4, 5, 3, and 5, respectively. Subsequently, the fine-tuning optimization is conducted and the optimal node is defined for each area among all neighbor nodes. Table 6 displays the values of $R M S_{v}$ and $R M S_{m}$ for the existing layout, the optimal layout, and the optimal layout after fine-tuning. The optimal fixture layout without fine-tuning has $23 \%$ and $12 \%$ improvements in variations and the mean deviation compared with the original layout. The variation has further improved by more than $7 \%$ after fine-tuning the locator positions in the locating areas.

\subsection{Verification of optimizations}

There are some uncertainties associated with meta-heuristic optimization algorithms in finding the global optimum. To overcome this problem, the optimization is repeated five times for each problem. Hence, obtaining similar solutions in all replications is considered an indication of the certainty in the results.

In the replication of optimization procedure for the first sample case, the optimal solution presented in Section 5.1 was obtained in three replications. The other
Table 5 The geometrical quality of sample case 1 for the existing and the optimal fixture layouts

\begin{tabular}{llc}
\hline Fixture layout & $R M S_{v}$ & $R M S_{m}$ \\
\hline The existing layout & 1.42 & 0.36 \\
The optimal layout without limits & 0.58 & 0.17 \\
The restricted optimal layout & 0.61 & 0.17 \\
Fine-tuned optimal layout & 0.56 & 0.16 \\
\hline
\end{tabular}




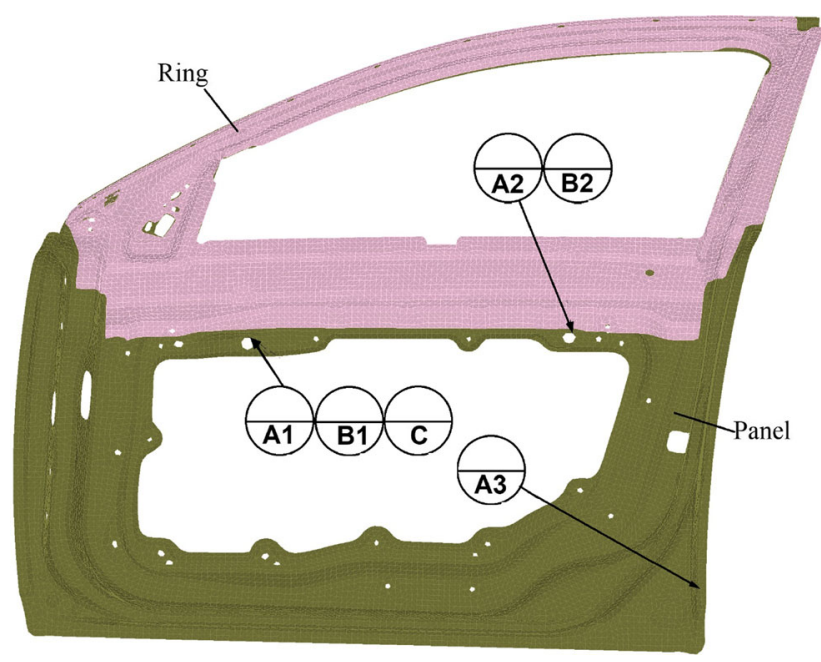

Fig. 11 Datum positions in sample case 2

two replications resulted in identical fixture layouts as the fixture layout presented in Section 5.1 for Water Channel. However, there are small differences in the number and location of additional locators for the other two parts. Table 7 lists a summary of the replication results compared with the results presented in Section 5.1.

Based on the results presented in Table 7, the fixture layout presented in Section 5.1 is selected as the global optimal fixture layout. The results also present that the fixture layout for Filler Piece plays a more important role than the other two parts. This is because all replications resulted in an identical fixture layout for this part.

The optimal fixture layout for the second sample case resulted in an identical fixture layout as it is presented in Section 5.3 in all five replications.

\section{Discussions}

The results demonstrate that the method presented can find quite robust fixture layouts for compliant sheet metal assemblies. This method can be applied to multi-station compliant sheet metal assemblies in a top-down design procedure. In this procedure, the locations of some locators in design of each fixture layout are already determined in the previous stations. The results of the first sample case evidence that this limitation may not arise a significant reduction in the possible improvements. The reason is that the total number of locators in a fixture layout for compliant assemblies is usually considerably more than the number of locators that are defined by the later stations. Therefore, having some locations fixed before the optimization does not affect the results significantly. Besides, the robustness of the fixture layouts in the subsequent stations has a greater influence on the geometrical quality of the final assembly.

The possible additional clamps in the fixture layout of the first and second sample cases were 30 and 25, respectively. However, the results evidence that the optimal fixture layout is not the one that has the maximum number of additional clamps. The optimum layouts contain only some of the possible additional clamps. In other words, adding additional clamps does not always improve the geometrical quality and robustness, and if they are not added optimally, they can reduce the geometrical quality of the assemblies. To verify this conclusion, the variations of each sample case when all possible additional clamps are added to the optimal fixture layout are also obtained for each sample case. The $R M S_{v}$ of the first and second sample cases for these fixture layouts are 2.76 and 2.04, respectively. These variations are significantly higher than the variations for the optimal and original fixture layouts of each case. Hence, adding additional clamps that are not in the optimal location and number has significantly reduced the geometrical quality of these sample cases.

The amount of improvements that can be achieved by fine-tuning is dependent on the size of defined candidate positions. If these areas are relatively larger, a higher improvement can be expected.

The elapsed time of optimization is very dependent on the elapsed time of variation simulations. A simulation for the first sample case takes about $20 \mathrm{~s}$. Consequently, the optimization time for the first sample case was around

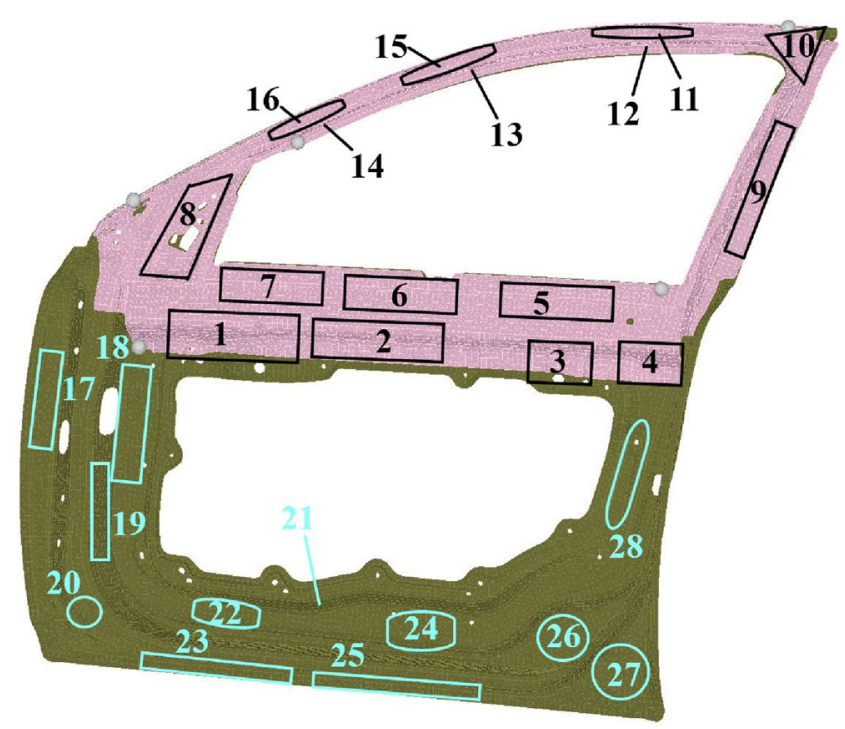

Fig. 12 Candidate locating areas in sample case 2 


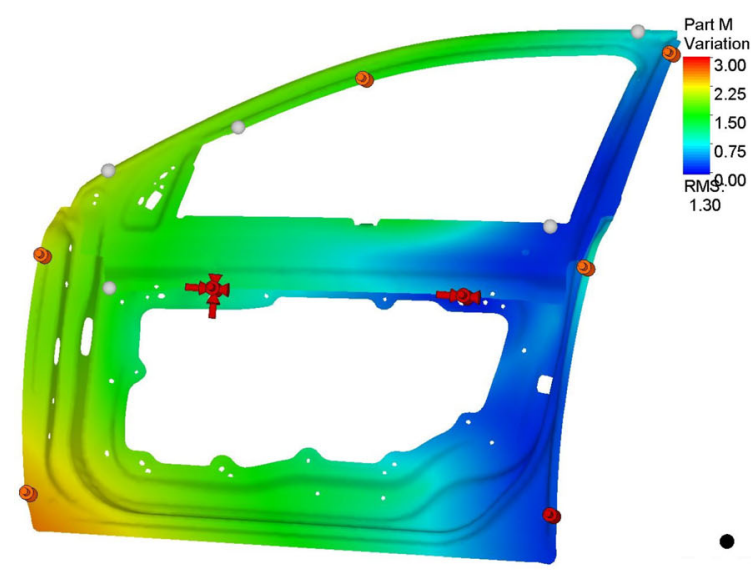

(a) Existing fixture layout

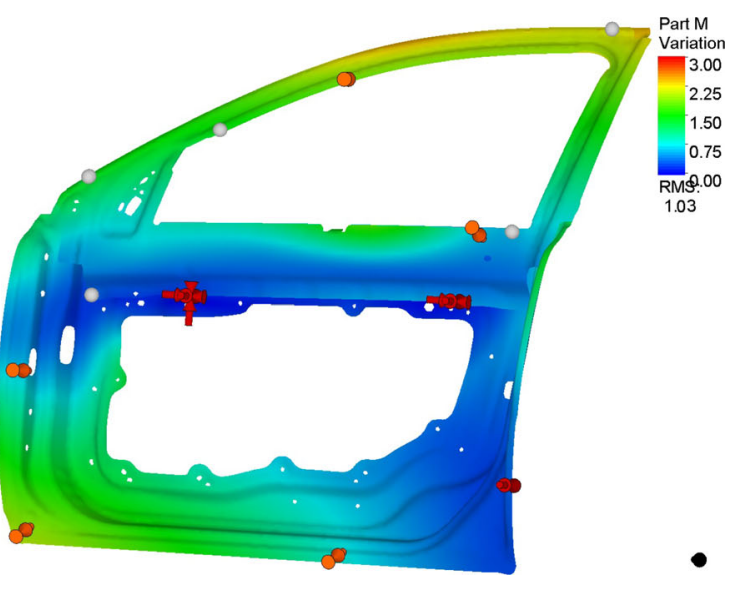

(b) Optimal fixture layout

Fig. 13 Existing and the optimal fixture layouts and color-coding of their variations in for sample case 2

$110 \mathrm{~h}$. The simulation time for the second sample case was $30 \mathrm{~s}$ that resulted in a total elapsed time of $160 \mathrm{~h}$.

\subsection{Future work}

The presented method in this paper was focused mainly on spot-welded sheet metal assemblies. This method can be developed in future studies for seam-welded assemblies. Moreover, utilization of surrogate functioning instead of conducting variation simulations in each function evaluation can be assessed in future research. Extension of this method for cases in which the parts can have more than one hole and slot, or they can be fixated without any hole or slot, can be investigated in future studies. Furthermore, general rules for fixture layout can be studied by applying the presented method and comparing the results for several different cases. The effects of utilizing the presented method in fixture design on the design process and requirement settings of each assembly and sub-assembly in BIW can be assessed in future studies. Design and optimization of fixture layouts for multi-station assemblies can be assessed by presenting proper sample cases that are assembled in several stations in another research study.

\section{Conclusions}

This paper presented a new method of fixture layout optimization for compliant assemblies, taking the effect springback and flexibility into consideration. The method encompasses optimizing all design parameters of a fixture layout, including the location and type of holes and slots for fixating each part of the assembly, the slot orientations, and the number and locations of additional clamps that overconstrain the parts. Moreover, a top-down design procedure was proposed for utilizing the presented method in fixture layout optimization of multi-station compliant assemblies.

The optimization process is divided into two stages. In the first stage, the feasible areas that practically can contain a locator position are defined. Then, these areas are divided into smaller areas and a node from each area is considered as the representative of that area. Thereafter, the optimal values of all design parameters are obtained. Subsequently, the representative node of each area is fine-tuned in the second stage of the optimization. If the size of each area is relatively large, the second stage can be repeated using a finer resolution of fine-tuning until the required accuracy is obtained.
Table 6 The geometrical quality of sample case 2 for the existing and optimal fixture layouts

\begin{tabular}{llc}
\hline Fixture layout & $R M S_{v}$ & $R M S_{m}$ \\
\hline The existing layout & 1.31 & 0.31 \\
The optimal layout & 1.03 & 0.26 \\
Fine-tuned optimal layout & 0.94 & 0.26 \\
\hline
\end{tabular}


Table 7 Results of five replications of optimization for the first sample case compared with results presented in Section 5.1

\begin{tabular}{lll}
\hline Repetitions & Differences & $R M S_{v}, R M S_{m}$ \\
\hline 3 times & No differences & $0.61,0.17$ \\
1 time & An extra clamp in area 2 of mounting plate & $0.65,0.17$ \\
1 time & Area 4 of Filler Piece is not clamped & $0.68,0.17$ \\
\hline
\end{tabular}

The percentage of improvements in variations in the finetuning stage was $8 \%$ for the first sample case and $7 \%$ for the second sample case. This improvement can be greater if the sizes of the candidate areas are larger compared to the presented sample cases. Accordingly, the fine-tuning stage can be neglected if the areas are small enough to provide the required accuracy in the optimal location of each locating point.

The following conclusions can be drawn from the results.

- The optimal fixture layouts of multi-station compliant assemblies can be determined by the presented method. Limiting the location of some locators in each station to the optimal positions of the later stations may not reduce the achievable geometrical quality of those stations.

- Compliant variation simulations should be utilized to optimize the fixture layouts to be able to consider overconstraining and springback into account. Moreover, determining the number and locations of clamps is possible only if the flexibility of assemblies is considered.

- Adding additional clamps does not always improve the geometrical quality and robustness. If their number and locations are not optimal, they can result in a lower geometrical quality of assemblies.

Acknowledgments This work was carried out in collaboration within Wingquist Laboratory and the Area of Advance Production at Chalmers within the project Smart Assembly 4.0, financed by The Swedish Foundation for Strategic Research. Their support is gratefully acknowledged.

Funding Open access funding provided by Chalmers University of Technology.

Open Access This article is licensed under a Creative Commons Attribution 4.0 International License, which permits use, sharing, adaptation, distribution and reproduction in any medium or format, as long as you give appropriate credit to the original author(s) and the source, provide a link to the Creative Commons licence, and indicate if changes were made. The images or other third party material in this article are included in the article's Creative Commons licence, unless indicated otherwise in a credit line to the material. If material is not included in the article's Creative Commons licence and your intended use is not permitted by statutory regulation or exceeds the permitted use, you will need to obtain permission directly from the copyright holder. To view a copy of this licence, visit http:// creativecommonshorg/licenses/by/4.0/.

\section{References}

1. Thornton AC (2004) Variation risk management: focusing quality improvements in product development and production, Wiley, Hoboken

2. Ceglarek D, Shi J (1995) Dimensional variation reduction for automotive body assembly 8:139-154

3. Söderberg R, Lindkvist L, Carlson J (2006) Virtual geometry assurance for effective product realization. In: Proceedings of $1^{\mathrm{ST}}$ nordic conference on product lifecycle management NordPLMÓ6, Gothenburg Sweden

4. Kim P, Ding Y (2004) Optimal design of fixture layout in multistation assembly processes. IEEE Trans Autom Sci Eng 1(2):133-145. https://doi.org/10.1109/TASE.2004.835570

5. Edholm P, Lindkvist L, Söderberg R (2012) Geometrical coupling analysis to reduce complete assembly line complexity. ASME Int Mech Eng Cong Expos 3:89-96. https://doi.org/10.1115/IMECE2012-85788

6. Jin J, Shi J (1999) State space modeling of sheet metal assembly for dimensional control. J Manuf Sci Eng 121(4):756-762. https://doi.org/10.1115/1.2833137

7. Phoomboplab T, Ceglarek D (2008) Process yield improvement through optimum design of fixture layouts in 3D multistation assembly systems. J Manuf Sci Eng Trans ASME 130(6):0610,051-06100,517. https://doi.org/10.1115/1.2977826

8. Cai W (2006) Robust pin layout design for sheet-panel locating. Int J Adv Manuf Technol 28(5-6):486-494. https://doi.org/10. 1007/s00170-004-2402-2

9. Zhaoqing T, Xinmin L, Zhongqin L (2009) Robust fixture layout design for multi-station sheet metal assembly processes using a genetic algorithm. Int J Prod Res 47(21):6159-6176. https://doi.org/10.1080/00207540802178091

10. Xie W, Deng Z, Ding B, Kuang H (2015) Fixture layout optimization in multi-station assembly processes using augmented ant colony algorithm. J Manuf Syst 37:277-289. https://doi.org/10.1016/j.jmsy.2014.08.005

11. Tyagi S, Shukla N, Kulkarni S (2016) Optimal design of fixture layout in a multi-station assembly using highly optimized tolerance inspired heuristic. Appl Math Model 40(11-12):61346147. https://doi.org/10.1016/j.apm.2015.12.030

12. Masoumi A, Shahi VJ (2018) Fixture layout optimization in multistation sheet metal assembly considering assembly sequence and datum scheme. Int J Adv Manuf Technol 95(9-12):4629-4643. https://doi.org/10.1007/s00170-017-1551-z

13. Camelio JA, Hu SJ, Ceglarek D (2004) Impact of fixture design on sheet metal assembly variation. J Manuf Syst 23(3):182-193. https://doi.org/10.1016/S0278-6125(05)00006-3

14. Liao X, Wang GG (2008) Simultaneous optimization of fixture and joint positions for non-rigid sheet metal assembly. Int J Adv Manuf Technol 36(3-4):386-394. https://doi.org/10.1007/s00170006-0827-5

15. Das A, Franciosa P, Ceglarek D (2015) Fixture design optimisation considering production batch of compliant Non-Ideal sheet metal parts. Procedia Manuf 1:157-168. https://doi.org/10.1016/j.promfg.2015.09.079 
16. Franciosa P, Gerbino S, Ceglarek D (2016) Fixture capability optimisation for early-stage design of assembly system with compliant parts using nested polynomial chaos expansion. Procedia CIRP 41:87-92. https://doi.org/10.1016/j.procir.2015.12.101

17. Lu C, Zhao HW (2015) Fixture layout optimization for deformable sheet metal workpiece. Int J Adv Manuf Technol 78(1-4):85-98. https://doi.org/10.1007/s00170-014-6647-0

18. Xing YF (2017) Fixture layout design of sheet metal parts based on global optimization algorithms. J Manuf Sci E-T ASME 139(10):1-10. https://doi.org/10.1115/1.4037106

19. Yu K (2019) Robust fixture design of compliant assembly process based on a support vector regression model. Int J Adv Manuf Technol 103(1-4):111-126. https://doi.org/10.1007/s00170-01903488-6

20. Asada H, By A (1985) Kinematic analysis of workpart fixturing for flexible assembly with automatically reconfigurable fixtures. IEEE J Robot Autom 1(2):86-94. https://doi.org/10.1109/JRA. 1985.1087007

21. Rong YK, Huang SH, Hou Z (2005) Chapter 6 - fixture modeling and analysis. In: Rong YK, Huang SH, Hou Z (eds) Advanced computer-aided fixture design. Academic Press, Burlington, pp 344-409. https://doi.org/10.1016/B978-012594751-0/50007-7

22. Liu SC, Hu SJ (1997) Variation simulation for deformable sheet metal assemblies using finite element methods. Journal of Manufacturing Science and Engineering, Transactions of the ASME 119(3):368-374. https://doi.org/10.1115/1.2831115

23. Camelio JA, Hu SJ, Marin SP (2004) Compliant assembly variation analysis using component geometric covariance. J Manuf Sci Eng 126(2):355-360. https://doi.org/10.1115/1.1644553

24. Camelio J, Hu SJ, Ceglarek D (2003) Modeling variation propagation of Multi-Station assembly systems with compliant parts. J Mech Des 125(4):673. https://doi.org/10.1115/1.1631574

25. Dahlström S, Lindkvist L (2007) Variation simulation of sheet metal assemblies using the method of influence coefficients with contact modeling. J Manuf Sci Eng 129(3):615-622

26. Wärmefjord K, Lindkvist L, Söderberg R (2008) Tolerance simulation of compliant sheet metal assemblies using automatic node-based contact detection. In: ASME 2008 International Mechanical Engineering Congress and Exposition, pp 35-44
27. Tabar RS, Wärmefjord K, Söderberg R (2019) A new surrogate model-based method for individualized spot welding sequence optimization with respect to geometrical quality. The International Journal of Advanced Manufacturing Technology, pp 1-14. https://doi.org/10.1007/s00170-019-04706-x

28. Lorin S, Lindkvist L, Söderberg R, Sandboge R (2013) Combining variation simulation with thermal expansion simulation for geometry assurance. J Comput Inform Sci Eng 13(3):031,007. https://doi.org/10.1115/1.4024655

29. Camuz S, Lorin S, Wärmefjord K, Söderberg R (2019) Nonlinear material model in part variation simulations of sheet metals. J Comput Inform Sci Eng 19(2):021,012. https://doi.org/10.1115/1.4042539

30. Babu M, Franciosa P, Ceglarek D (2019) Spatio-Temporal Adaptive Sampling for effective coverage measurement planning during quality inspection of free form surfaces using robotic 3D optical scanner. J Manuf Syst 53:93-108. https://doi.org/10.1016/j.jmsy.2019.08.003

31. Franciosa P, Gerbino S, Patalano S (2011) Simulation of variational compliant assemblies with shape errors based on morphing mesh approach. The Int J Adv Manuf Technol 53(1):4761. https://doi.org/10.1007/s00170-010-2839-4

32. Schleich B, Wartzack S (2015) Approaches for the assembly simulation of skin model shapes. Comput Aided Des 65:18-33. https://doi.org/10.1016/j.cad.2015.03.004

33. Rezaei Aderiani A, Wärmefjord K, Söderberg R (2020) An improved phenotype-genotype mapping for solving selective assembly problem using evolutionary optimization algorithms. Journal of Computing and Information Science in Engineering 20(6). https://doi.org/10.1115/1.4047241, 061010

34. Bäck T (1996) Evolutionary Algorithms in Theory and Practice. Oxford University Press

35. Eshelman LJ, Schaffer JD (1993) Real-Coded Genetic Algorithms and Interval-Schemata. pp 187-202

36. Patil V, Pawar D (2015) The optimal crossover or mutation rates in genetic algorithm: a review. Int J Appl Eng Technol 5(3):3841

Publisher's note Springer Nature remains neutral with regard to jurisdictional claims in published maps and institutional affiliations. 